\title{
Estimation of Fruit Locations in Orchard Tree Canopies Using Radio Signal
}

\section{Ranging and Trilateration}

\author{
Rajkishan Arikapudi ${ }^{\mathrm{a}}$, Stavros G.Vougioukas ${ }^{\mathrm{a},{ }^{*}}$, Francisco Jiménez- Jiménez ${ }^{\mathrm{b}}$, Farangis Khosro \\ Anjom $^{\mathrm{a}}$
}

\section{Shields Ave., Davis, CA 95616, U.S.A.}

${ }^{\mathrm{a}}$ Department of Biological and Agricultural Engineering, University of California, Davis.One

${ }^{\mathrm{b}}$ Department of Rural Engineering, Universidad de Córdoba. Ctra. Nacional IV, km. 396, 14014

Córdoba, Spain.

${ }^{*}$ Corresponding author:E-mail address: svougioukas@ucdavis.edu.

\section{Abstract.}

The development of novel robotic harvesters could benefit significantly from a model-based design approach,in which harvesting performance metrics--such as fruit reachability and average pick-andplace cycle--are calculated via simulation, and are used to guide mechanical design. The actual spatial distributions of fruits on orchard trees are necessary for such an approach. Reported methods for measuring the locations of all fruitsrequire several minutes per fruit, and,consequently, have been used only for very small numbers of trees. The novel method presentedutilizes high-frequency radio signals and trilateration to measure the locations of all fruits in canopies,at speeds that are significantly higher than those of existing methods. More specifically, a fruit picker wears gloves on which an antenna has been attached. A mobile trailer carries four radio beacons that measure and log their distances from the antenna on each glove, every time a fruit is grasped to be picked. The coordinates of each glove are computed with respect to a coordinate frame attached to the trailer, 

inclinometer on the trailer are used to compute georeferenced fruit coordinates. Data were collected for 32,193 fruits in eight California pear and cling peach orchards. The measurement rate varied from approximately 8 to 12 fruits per minute, with an average of 10.8 , which is a magnitude faster than existing reported methods. In open space, the root mean square error between the estimated and true distance (DRMS) in the system's measurement volume was measured to be $10.3 \mathrm{~cm}$. The error's $90^{\text {th }}$ percentile (R90) was $13.1 \mathrm{~cm}$. In the periphery of and inside canopies, these errors were calculated via Monte Carlo simulation to be equal to $15.7 \mathrm{~cm}$ and $24.9 \mathrm{~cm}$ respectively. The horizontal accuracies (across and along the row), andthe vertical accuracy were $9.6 \mathrm{~cm}, 4.3 \mathrm{~cm}$ and $5.7 \mathrm{~cm}$ respectively. The corresponding worst-case relative accuracies were $2.7 \%, 1.6 \%$, and $3.4 \%$, and were calculated by dividing each accuracy componentby the distance between the fruits that were as far away as possible from each other along the corresponding axis. Finally, fruit positionstatistics,such asfruit elevation and horizontal distance from the row centers were computed and reported for a set of pear trees.Such data can be very useful for growers and for model-based design of harvesting machinery.

\section{INTRODUCTION}


performance in the orchard will depend on the complex interrelationships among orchard layout, tree canopy structure, spatial fruit distribution, harvester kinematics and dynamics, fruit detection and localization, and actuator control. For this reason, performance is evaluated using physical prototypes in orchards during the short harvesting season. This fact renders the iterative process of designing, building, testing and modifying fruit-harvestersexpensive and slow.

The development of novel robotic harvesters could benefit significantly from a model-based design approach, in which harvesting performance metrics are estimated by simulating the parts of the harvesting operation that are essential and meaningful for design purposes.A major goal of modelbased design would be to guide toward a mechanical design that maximizes performance in terms of fruit kinematic reachability and average pick-and-place cycle for trees of certain architectures. This goal can be achieved by assuming perfect perception and control. In other words, predicting harvesting performance for mechanical design should incorporate the constraints imposed by the tree geometries and fruit positions, but not the constraints that may arise due to poor fruit visibility, underpowered actuation, or non optimal control. The use of model-based approaches for robotic harvester design and optimization has been very limited. Edan et al., (1991) modeled a specific robot arm type and measured fruit locations on a few small trees, in order to calculate optimal picking sequences for the robot, and to study the effect of link masses on maximum throughput. Sivaraman and Burks (2006; 2007) used canopy volume estimates to perform robot manipulator kinematic design, with maximum fruit reachability as a design criterion. Van Henten et al., (2009) used workspace and manipulator specifications stemming from cucumber harvesting operations to optimize the kinematic structure, and link parameters of a three-link robot arm.

This paper focuses on the measurement of spatial distributions of all fruits on trees. Branches certainly affect fruit accessibility and harvester throughput (and, consequently, optimal mechanical design); therefore, their geometrieswould need to be incorporated, too. Measuringthe locations of all fruits on trees can be a very time-consuming and expensive process. The first documented attempt to 
mapfruit spatial distributions on trees was by Schertz and Brown (1966), who adopted a cylindrical coordinate system and measured the coordinates of citrus fruits. More specifically, the longitudinal axis of the coordinate system was oriented vertically and collinearly with the trunk of the tree. The height of each fruit was measured by lowering a plumb bob on a calibrated tape. The point of intersection of the plumb bob with a polar coordinate board at ground level gave both the angular and the radial positions of each fruit. This approach was very time-consuming, and required onetotwo days to map all the fruits on a single tree, depending on yield and tree size. Measurement accuracy could not be assessed, but it obviously depended on user skill, because branches might also cause the tape to deflect and deviate from a straight vertical line. Other researchers, such as Juste and Lee and Rosa, have also used this simple approach (Juste, et al., 1988; Lee and Rosa, 2006).

Smith et al., (1992) used a theodolite to record the three-dimensional coordinates of kiwi fruits on vines and sample the geometric structure of the vines themselves. The accuracy of the readings was reported to be within millimeters of the position of each point. This method was limited, in that it required unobstructed Line-of-Sight (LOS) between each fruit and the theodolite. This, however, is impractical for trees with dense canopies and fruits hanging deep inside them. Also, fruit position measurement was slow: each fruit required approximately $60 \mathrm{~s}$, resulting in an effective rate of one vine per one-to-two days, depending on yield and vine size. Smith and Curtis (1996) used a threedimensional position tracking system (PolhemusFastTrak ${ }^{\mathrm{TM}}$ ) to digitize tree structure and measure fruit locations. This system used a low frequency magnetic field emitted by a transmitter to measure the 3-D coordinates of the tip of a stylus; coordinates were relative to a coordinate system defined by the transmitter. The RMS static accuracy of the system in the absence of metal objects is approximately $1 \mathrm{~cm}$, within $1 \mathrm{~m}$ from the transmitter. The system's operational radius was limited to approximately $1.5 \mathrm{~m}$; digitizing large trees was made possible by re-locating the transmitter to various positions, while keeping at least three points from the previous position within range. As a result, an appropriate coordinate transformation matrix can be computed. Data collection without moving the 
transmitter was reported to take place at approximately 10 points per minute. However, digitizing 1300-1500 points on a larger kiwi vine with this system required 10-12 hours.

Edan et al., (1991) measured fruit locations on twenty orange trees using a robotic manipulator on a mobile platform as digitizer. The data were collected manually, by placing the arm tip at each fruit on the tree, and storing the readings of each joint encoder at this position on a computer. Furthermore, the exact location of each fruit was calculated via the robot's forward kinematics. Accuracy and data collection rate were not reported. Relocating the robot tip - and possibly the platform -to reach each new fruit position should have required non-trivial effort, resulting in low collection rates.Also, larger trees would require a large robot, whilebranches could interfere with the robot links, thus rendering the task very difficult.

In contrast to manual methods, vision-based approaches use multiple cameras and points of view to detect and triangulate fruits from images. Takahashi et al. (2002) used stereovision to measure the range of apple fruits. The camera's position and Euler angles could be combined with the range to compute $3 \mathrm{D}$ fruit location. The range estimation errors were reported to be $\pm 5 \%$ (average of $-1.3 \%$ and standard deviation of $3.5 \%$ ) at a distance range of $1.7 \mathrm{~m}$ to $5.2 \mathrm{~m}$ for non-overlapping fruits. Fruit localization has also been performed in yield estimation systems, such as the one developed by Wang et al., (2013), in order to avoid double counting of fruits in sequences of images. The authors do not report the position accuracy of the system, although they mention that fruits closer to $5 \mathrm{~cm}$ are counted as a single fruit. Here, it is important to note that their goal was yield mapping and not fruit localization. The major problem with vision-based approaches is that many fruits - especially those deeper into the canopy - are occluded by other fruits, leaves, or branches, and are not visible from the camera's viewpoints. For this reason, they cannot be localized.

In this paper, a novel method is presented, which utilizes Ultra-Wide-Band (UWB) high-frequency radio signals and trilateration to measure the locations of allfruits in tree canopies, while the fruits are being picked.UWB uses short pulse radio frequency waveforms over a large bandwidth $(\mathrm{GHz})$ to 
measure precisely the time-of-flight of a signal between a transmitter and a receiver. Trilateration combines the measured ranges into an estimated position. UWB radios have been proposed for localization in work environments associated with construction(Cheng, 2011) and mining (Chehri et al., 2009).To the authors' best knowledge, the use ofUWB technology for localizing points close to, or inside, tree canopies has not been reported in the literature. Results from data collection in several orchards with different tree architectures are presented. These results establish that an average throughput of the system is approximately 10.8 fruits per minute, which is at least one order of magnitude faster than existing reported methods.

Another contribution of this work is the investigation of the effects of foliage on trilateration accuracy. It is known that the water contained in vegetation attenuates radio signals, and that signal reflection on the ground, as well as scattering at tree branches introduce multipath effects (Vougioukas et al., 2013) that reduce UWB ranging accuracy. A novel trilateration error assessment method is also presented, which uses the statistical distributions of range errors to estimate the trilateration error inside foliage. The method is validated by means of experimental results, which are used to characterize the accuracy of the measuring system. Finally, as an example of using the fruit position data, some spatial statistics that could be relevant to machine design are calculated and presented.

\section{Materials ANd Methods}

In this section, the hardware used in the experiments is presented,a description of the fruit position measurement method is offered,and mathematical procedures for the estimation of positions and position errorsare described in detail.

\subsection{HARDWARE}

The radio ranging transmitting and receiving units (RTRUs) used in this research are the PulsON 400 Ranging and Communications Modules (P400 RCM) (Time Domain, Inc, Huntsville, AL, USA). The P400 RCM is an Ultra Wideband (UWB) radio transceiver that measures the distance between the center-points A and B of two P400 RCM antennas (Figure 1). The units measure this distance 


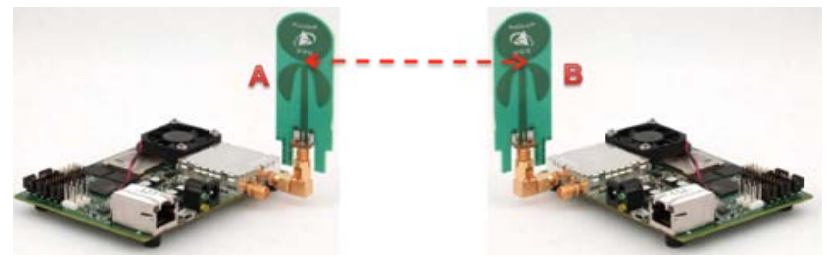

Figure 1. A pair of UWB radios: Unit A measures the distance (red dotted line) between center points of antennas A and B.

The units operate in the band of $3.1 \mathrm{GHz}$ to $5.3 \mathrm{GHz}$, with a center frequency at $4.3 \mathrm{GHz}$. In a static scenario (when units are not moving) with an unobstructed LOS, the specifications for the range measurement precision (standard deviation) is $2.3 \mathrm{~cm}(0.9 ")$, and for accuracy (bias) is $2.1 \mathrm{~cm}$ $\left(0.83^{\prime \prime}\right)$. The units were configured to use seven pulses per data symbol $(\mathrm{PII}=7)$, which resulted in a maximum LOS range of $125 \mathrm{~m}(410 \mathrm{ft})$, and a measurement period of $20 \mathrm{~ms}$.

A mobile trailer (Figure 2) carried four identical RTRUs and their corresponding antennas on top of tall aluminum poles(Figure 3); the trailer RTRUs are also referred to astrailerbeacons. The antenna coordinates with respect to the trailer's coordinate frame are shown in Figure 2. The poles carrying the beacons were placed as far apart as possible on the trailer, under the constraint that they should not interfere with tree branches extending out into the orchard rows. The reasoning behind this choice is that the bigger the distance between beacons along an axis is, the better the localization accuracy along this axis will be(Manolakis, 1996). For the same reason, one beacon was placed much lower than the others. 


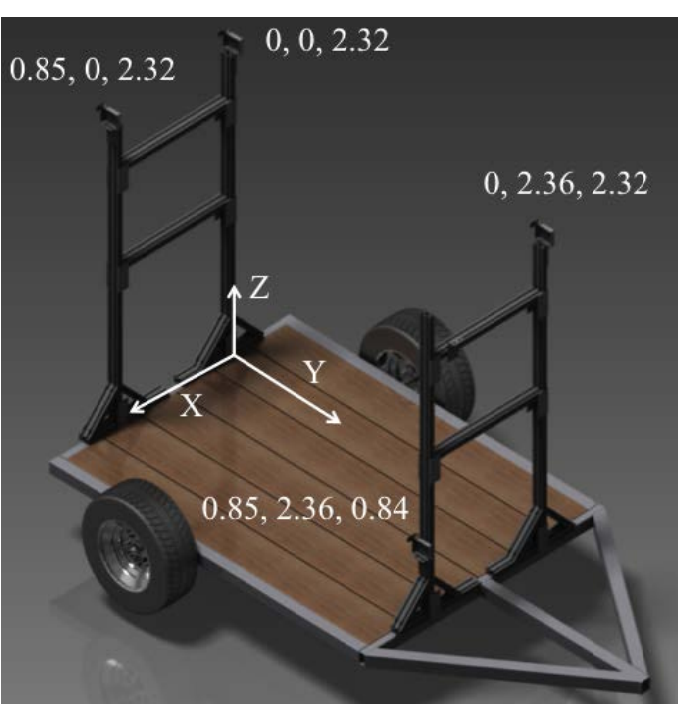

Figure 2. CAD model of the trailer used to carry the four radio beacons.

170

171

172 173

180

181

182

183

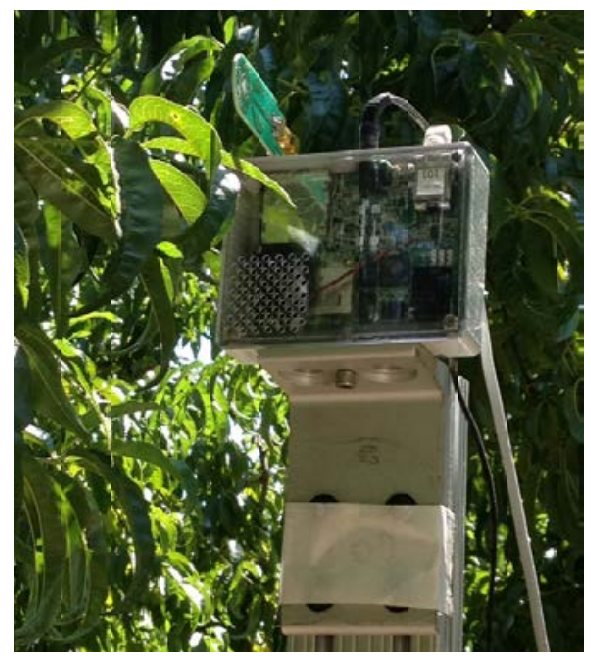

Figure 3. A radio beaconplaced at the top of a pole on the trailer. Poles are made of T-slotted aluminum extrusion rails.

The Trimble AgGPS 542 GNSS/RTK GPS System was used to record in real-time the georeferenced location of the trailer with centimeter-level accuracy. The Ag542 Rover GPS on the trailer (Figure 4) received corrections from the Ag 542 Mobile Base Station GPS, using 900Mhz radiomodems integrated in the receivers. 


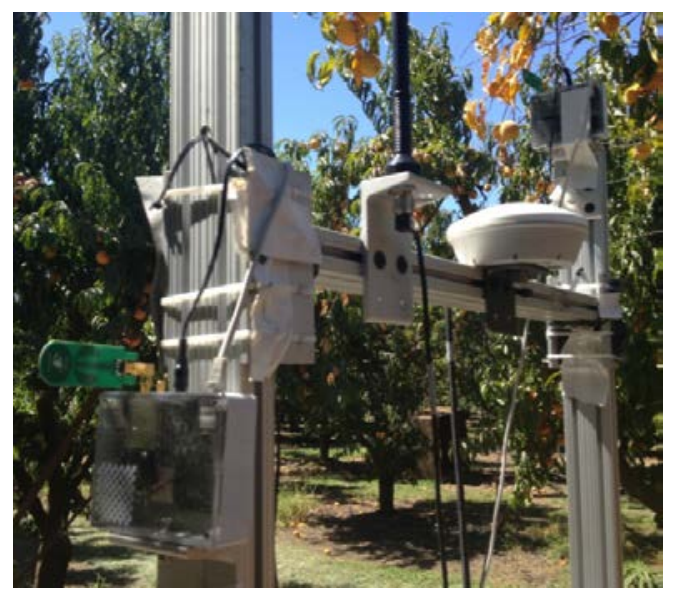

Figure 4. RTK GPS receiver and modem antenna on the trailer provide accurate positioning for the trailer beacons.

A wireless attitude and heading sensor by YEI Technology, Inc. (Portsmouth, OH, USA) was used to measure the trailer's roll, pitch, and heading angles. The sensor has $\pm 1^{\circ}$ orientation accuracy for dynamic conditions, and repeatability better than $0.008^{\circ}$. The sensor outputs filtered absolute orientation angles in Euler format (pitch/roll/yaw). The computed order of rotation for the Euler angles is XZY. Therefore, the total Euler rotation matrix, $R$ is composed of three rotation matrices, multiplied as $R=R_{y} R_{z} R_{x}$. The three angles are reported by each sensor as $\psi, \theta, \varphi$, where pitch $(\psi)$ is rotation about the $\mathrm{X}$-axis (looking towards $\mathrm{X}$; clockwise is positive), heading $(\theta)$ is about the Y-axis (looking towards $\mathrm{Y}$; clockwise is positive), and roll $(\varphi)$ is about the Z-axis (looking towards Z: clockwise is positive). Using GPS and angles, the 3D positions of the trailer beacons could be computed with respect to the world frame; Universal Transverse Mercator (UTM) coordinates were used for the projected coordinates.

The CST/berger 305R Electronic Reflector-less Total Station was used to locate the true positions (ground truth) of the trailer and glove beacon antennas in all experiments. The device uses a laser beam to locate a target surface and to report its angular position and coordinate position with respect to its own frame. The device has 5-second angle accuracy at a sighting distance of $100 \mathrm{~m}$, which is equivalent to an accuracy of $2.5 \mathrm{~mm}$ in distance measurement. In all experiments, the operating range of the system was confined to $10 \mathrm{~m}$, which translates into an accuracy of $0.2 \mathrm{~mm}$. 


\subsection{Fruit Position Measurement Method}

The fruit position measurements were made during manual harvesting with bags and ladders. In each orchard, a number of trees along a row weretagged, and each tree wasgiven a unique identification (ID) code. In the orchard row, the trailer was placed next to the tree(s) to be picked, having the tree row at its right side. A picture of the trailer positioning relative to a tree-row from actual experiments is shown inFigure 5 .

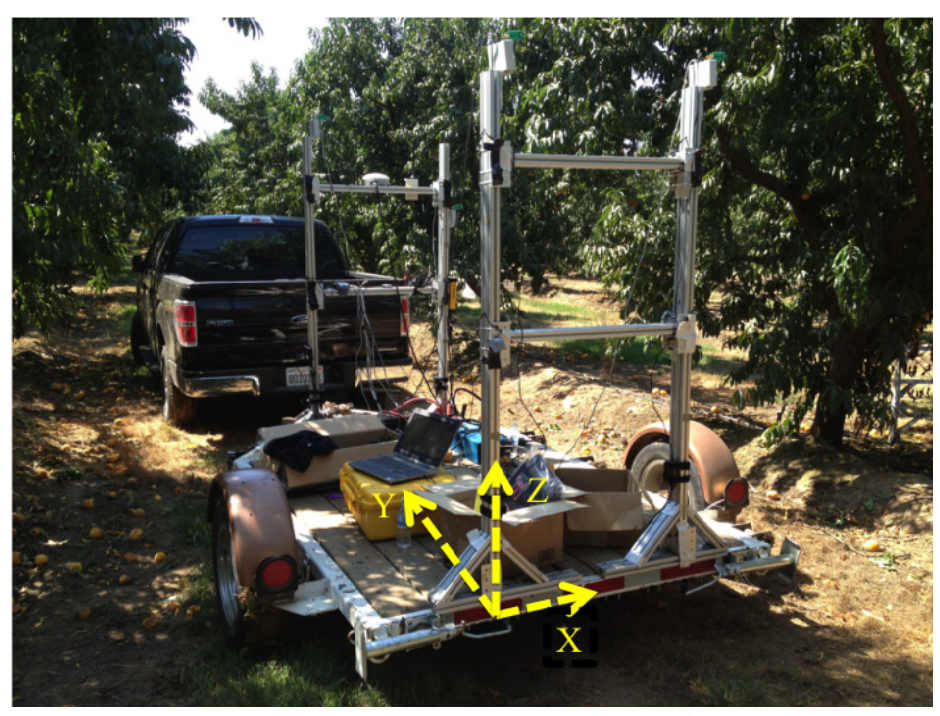

Figure 5. Photoof the trailer between tree-rows; it was taken during system deployment in an orchard.

The length of the system's intended measurement volume along the row (Y axis) was $4.5 \mathrm{~m}$, its width ( $\mathrm{X}$ axis, into the canopy)was $2.4 \mathrm{~m}$, and its height ( $\mathrm{Z}$ axis) was $4.5 \mathrm{~m}$. The system's measurement volumewas intended to cover the left half of the volume ofeach measured orchard tree; the right half volume is picked with the trailer placed in the right-adjacent row. The reason behind this choice is that fruit picking was also limited tohalf the tree volume, on the side of the row that the trailer was positioned, in order to reduce the amount of foliage and the resulting signal attenuation, scattering and diffraction between the glove antenna and the beacon antennas.

A pickerwasinstructed to pick all the fruits from the lefthalves of the tagged trees inside the trailer's measurement volume.After finishing, the trailer was moved forward to the next unpicked trees in the $+Y$ direction. The picker carried one small Radio Ranging Transmitter and Receiver Unit (RTRU) on 
217 the RTRU on the belt (two antennas per RTRU). The pickerwasinstructed to pick only one fruit at a 218 time, without displacing the branch of the fruit, in order not to change its original location. Also, the 219 picker was instructed to grasp the fruit in a manner that the antenna would face the trailer beacons, 220 i.e., the vector normal to the picker's palm would face toward the negative X-axis.

221

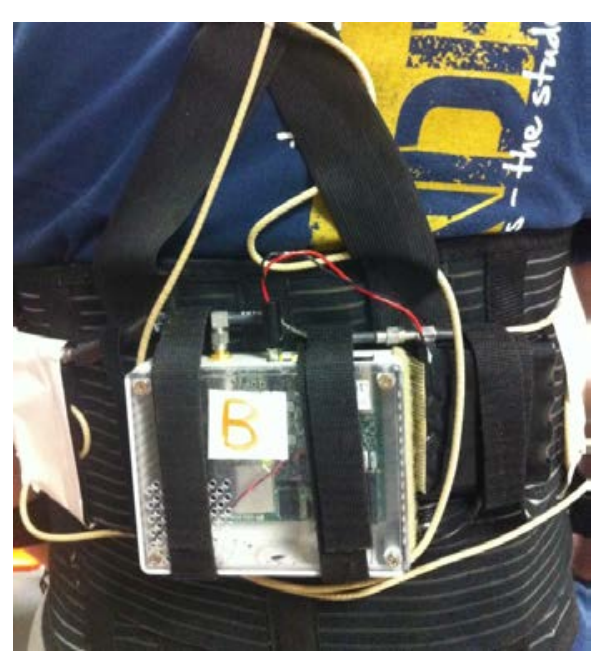

Figure 6. Ranging transmitter - receiver unit (RTRU) attached with Velcro on a belt worn by the picker.

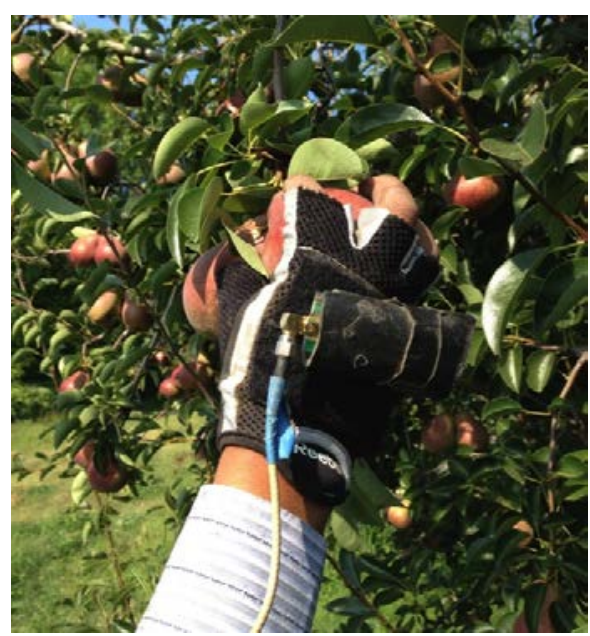

Figure 7. The mobile beacon antenna was placed in a protective enclosure (flexible PVC tube) that was attached to the picker's glove.

Every time the picker grasped a fruit in order to detach it from a tree, a human observer standing

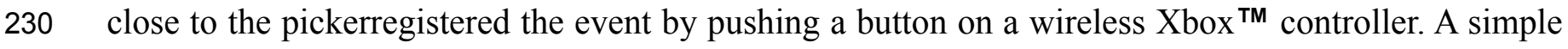


"protocol" was established to ensure the synchronization of the fruit-grasp event and the recording of

232

233

234

235

236

237

238

239

240

241

242

243

244

245

246

247

248

249

250

251

252

253

254 the four mobile-to-trailer beacon antenna ranges. First, the picker was instructed to shout "Aye" upon fruit grasping. Secondly, the observer verified visually that the picker was grasping the fruit, pushed the button to initiate the recording of the ranges, waited to feel the controller vibrate upon completion of range recording (400 ms for four ranges - see next paragraph), and then shouted "OK". Last, the picker detached the fruit upon hearing "OK" from the observer. The protocol was used consistently; however, it is possible that, on some occasions, the picker's hand was not still, or in full contact with the fruit during the $400 \mathrm{~ms}$ interval of range recording. In such cases, the respective estimated fruit positions would contain errors that are difficult to model. Given the visual check by the observer and the short time duration of range recording, such errors are expected to be infrequent and minor; for this reason, they may not impact substantiallythe overall accuracy of the system.

Custom-written data collection software polled all sensors, and logged their readings together with the ID of the tree being harvested, the ID of the hand used (1 for left / 2 for right), and a unique timestamp for the fruit-grasp event. The GPS polling rate was kept low, at $1 \mathrm{~Hz}$, because the trailer wasstatic while the worker picked fruits. The attitude sensor was polled at $10 \mathrm{~Hz}$. Each beacon was polled every $20 \mathrm{~ms}$. Each recorded range was calculated as the median value of five consecutive range measurements, in order to reject outliers. Hence, a new filtered range update was available for each beacon, approximately every $100 \mathrm{~ms}$. Onelaptop was used for all data collection, and, therefore, all events were time-stamped using the same real-time clock.

After all fruitshad been collected, the 3D georeferenced coordinates of each picked fruit wereapproximated by the coordinates of the antenna on the hand of the picker, when the hand grasped the fruits. These coordinates wereestimated via trilateration-- a procedure that uses the measured ranges to compute an estimated position. 


\subsection{Trilateration of The Mobile Beacon Antenna}

Let the coordinates of the $j$ th trailer beacon with respect to the world frame be $\left(b x_{j}, b y_{j}, b z_{j}\right)$. Their calculation is derived in the Appendix. Each time a fruit is grasped by the worker, the timestamp of the "fruit-grasp" event is recorded together with a left or right hand flag, and an identification number for the tree that the fruit is picked from. Let $\mathbf{p}_{i}=\left[x_{i}, y_{i}, z_{i}\right]$ be the real coordinates of the glove antenna with respect to the world frame (measured with the Total Station), and $d_{i j}$ be the true (geometrical) distance between the $j$ th trailer beaconand $\mathbf{p}_{i}$ :

$$
d_{i j}=\left(\left(x_{i}-b x_{j}\right)^{2}+\left(y_{i}-b y_{j}\right)^{2}+\left(z_{i}-b z_{j}\right)^{2}\right)^{1 / 2}
$$

Due to multipath or intense signal attenuation conditions, the estimation of TOA (and subsequently of range) of the direct pathbetween beacon $j$ and point $\mathbf{p}_{i}$ will typically contain a random error.If this error is $\varepsilon_{i j}$, then the measured ranger $r_{i j}$ is: results in the optimal position estimation (Navidi et al., 1998):

$$
\left(x_{i}^{t}, y_{i}^{t}, z_{i}^{t}\right)=\underset{x_{i}, y_{i}, z_{i}}{\arg \min } \sum_{j=1}^{4}\left(r_{i j}-d_{i j}\right)^{2}
$$

The position coordinates of each picked fruit are approximated by the coordinates $\left(x_{i}^{t}, y_{i}^{t}, z_{i}^{t}\right)$ of the glove antenna on the surface of the fruit. All calculations were performed using Matlab R2013a (MathWorks, Natick, MA, USA); equation(3) was solved using the fminsearch() Matlab optimization function.

\subsection{ERROR METRICS}

Let the $3 \mathrm{D}$ distance error between the trilaterated position $\mathbf{p}_{i}^{t}$ and the true position of the glove antenna at point $\boldsymbol{p}_{i}$ be: 
Let the distance error parallel to the orchard tree rows (y-axis) be $e_{y i}$, the error perpendicular to the tree rows be $e_{x i}$, and the error along the vertical axis be $e_{z i}$; these errors are defined as:

$$
e_{x i}=x_{i}^{t}-x_{i}, e_{y i}=y_{i}^{t}-y_{i}, e_{z i}=z_{i}^{t}-z_{i}
$$

Given a set of $N$ measurements, the system's accuracy was characterized using a number of metrics. The first accuracy metric is the Distance Root Mean Square error (DRMS):

$$
D R M S=\sqrt{\frac{1}{N} \sum_{i=1}^{N} e_{i}^{2}}
$$

The advantage of this metric is that it is a radial error; in other words, it is invariant to the rotation of the axes used to describe the error. However, in an orchard setting, it is important to distinguish between errors along the tree rowaxis, and errors perpendicular to it. Therefore, three additional metrics are computed--mainly, the average absolute errors in the $x$, yand zdirections:

$$
A B S E x=\frac{1}{N} \sum_{i=1}^{N}\left|e_{x i}\right|, A B S E y=\frac{1}{N} \sum_{i=1}^{N}\left|e_{y i}\right|, A B S E z=\frac{1}{N} \sum_{i=1}^{N}\left|e_{z i}\right|
$$

Two disadvantages of the above metrics are that isolated large outlier errors have a large effect on them, and that the metrics are not tied to a specific probability level. Therefore, the $90^{\text {th }}$ percentile or R90 error metric is also computed based on the $3 \mathrm{D}$ distance error,e. This metric is equal to the radius of a sphere centered at the true position, containing the position estimate with a $90 \%$ probability.

\subsection{Trilateration ERROR ASSESSMENT METHOD}

The trilateration error at each antenna location $p_{i}$ inside the measurement volume depends on: a) the relative geometrical arrangement of the mobile antenna and the fixed beacon antennas, i.e., $\mathbf{p}_{i}$ and ${ }^{W F} \mathbf{b}_{j}, j=1,2,3,4 ;$ and $\mathrm{b}$ ) the range errors $\varepsilon_{i j}$. Antenna placement causes the Dilution of Precision (DOP) effect: when position is computed via trilateration, the range errors are amplified by a factor that depends on the relative arrangement of the antennas (Manolakis, 1996), which, in our case, varies 
over the measurement volume. In open space with unobstructed LOS range, radio signal path loss is not spatially uniform, and is typically larger close to the ground, because of wave reflection and resulting signal fading and multipath echoes (Saidi, et al., 2011; Sangodoyin et al., 2016). These factors can increase UWB range errors (Sahinoglu, et al., 2008). In the presence of foliage, signal attenuation is typically higher and varies with distance, even at relatively shorter ranges(Vougioukas et al., 2013). Hence, when foliage is present in the LOS, range errors are expected to be higher. Main reasons include: increased signal attenuation from the water in the vegetation, increased multipath due to signal diffraction and scattering on leaves, branches and fruits, and lower light speed through leaves and branches (light speed is used to convert TOA to range).

For the reasons described above, the trilateration error is expected to vary over the measurementvolume.In open space, it is possible to measure directly the trilateration error at points on a sampling grid that covers the measurement volume using a surveying instrument (Total Station). The trilateration error at any position can then be estimated by interpolating the errors from its neighboring grid points. However, surveying instruments require unobstructed LOS to the position being measured. Therefore, measuring the trilateration errorson a sampling grid inside tree canopies is practically impossible, mainly because of foliage that blocks LOS. Furthermore, experimentally measured open-space trilateration errors cannot be used as "reasonable" estimates of trilateration errors for locations inside canopies, because the corresponding range errors exhibit different 319 behaviors.

This challenge is addressed by introducing a trilateration error assessment method thatutilizesMonte Carlo simulation to estimate trilateration errors based on measured range error statistics.Essentially,range errors from experimentally derived range error distributions propagate via the trilateration equation (3) to calculate trilateration errors over a grid of points in the measurement volume. Error propagation via Monte Carlo simulation is a well-established technique in metrology (JCGM 101:2008)that has been used extensively in various domains (e.g., Cox et al., 2002). The steps 
Sampling Step:Generate $K$ sets $\mathrm{H}_{k}(k=1, . ., K)$ of points, each containing $M$ points. These sets create a sampling distribution of points $\mathbf{p}_{i}$ that sample adequately the measurement volume;

Range Error Characterization Step:Estimate experimentally the probability distribution $\mathrm{P}_{k}(\varepsilon)$ of range errors $\varepsilon_{i j}$ from allbeacons to all points $\mathbf{p}_{i} \in \mathrm{H}_{k}$;

The application of this method to the fruit-position measuring system was validated in open space, where direct trilateration error measurements were available. Then, the probability distribution $\mathrm{P}_{k}(\varepsilon)$ of range errors in foliage were estimated (Sampling and Range Error Characterization Steps), and the Simulation Step of the above method was executed to predict trilateration errors for sets $H_{k}$, and the entire measurement volume in foliage. The experimental design of the measurement campaigns and the results will be presented in the following sections. 
It has been observed that trilateration error - in particular, the elevation component -is larger at

364 points closer to the ground (Saidiet al., 2011). Main reasons include wave ground reflections, and 365 resulting signal fading and multipath echoes. Therefore, five $(K=5)$ sets $\mathrm{H}_{k}$ of pointswere defined that correspond to five planes at increasing heights equal to $0.91(\mathrm{k}=1), 1.83(\mathrm{k}=2), 2.74(\mathrm{k}=3), 3.66(\mathrm{k}$

$367=4)$ and $4.57 \mathrm{~m}(\mathrm{k}=5)$. These correspond to approximately 3, 6, 9, 12 and $15 \mathrm{ft}$.These planes are 368 shown in Figure 8.

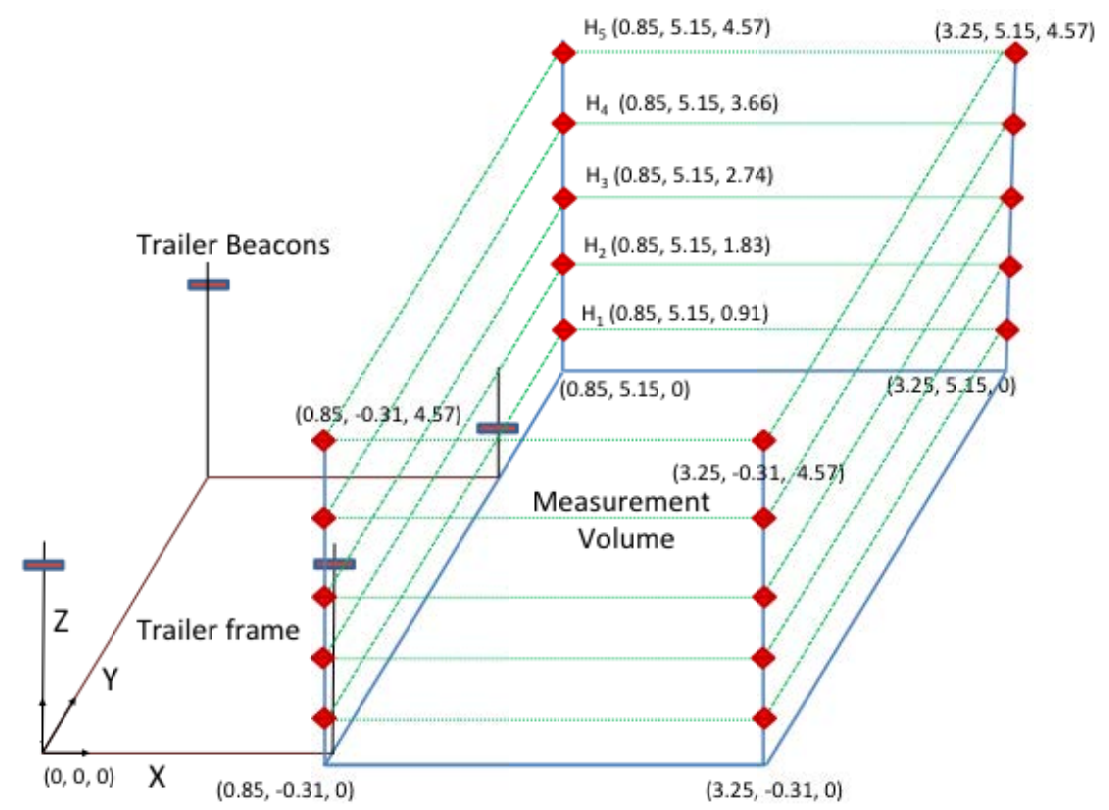

362

Figure 8: The system's measurement volume was sampled at five horizontal planes $\mathbf{H}_{k}$ of increasing heights.

Each plane contained $M=24$ points arranged in a uniform gridof size approximately equal to 0.6 $\mathrm{m} \times 0.9 \mathrm{~m}(2 \times 3 \mathrm{ft}$. $)$ A total of $K \times M=120$ points were used to sample the measurement volume. As an example, the points on the grid in $\mathrm{H}_{1}$ are shown as star symbols in Figure 9. 


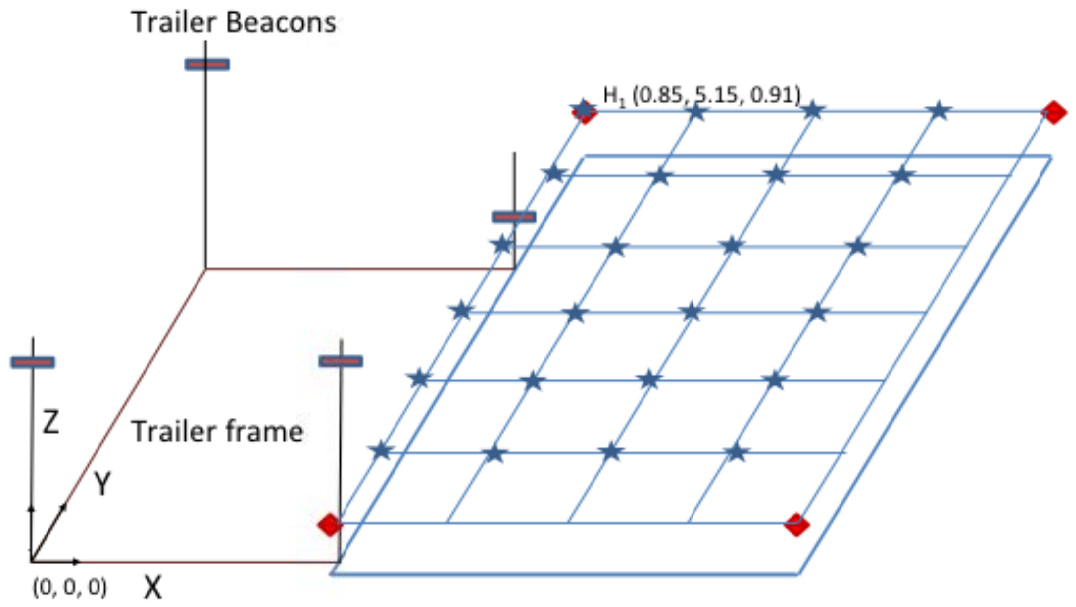

Figure 9: Illustration of measurement points forming a uniform grid on plane $\mathrm{H}_{1}$ of the measurement volume.

\subsection{Range ERror Measurement IN Open SPaCe}

In open space, it is possible to measure range errors at all points of the sampling grid. Four RTRUs on the trailer frame acted as known-position fixed beacons. A five-meter-long T-slotted aluminum extrusion rail was used as a mobile pole, and was placed at the $x-y$ ground coordinates of each 'column' of sampling points. At each position of the pole ( $M$ in total), an RTRU that acted as a mobile beacon was placedon the pole, and raised to each of the $K$ different heights defined by the planes. The coordinates of all beacon antennas were measured using the Total Station.Fixed-to-mobile beacon antenna distanceswere to be measured at all grid points. For each grid point $\mathbf{p}_{i}$ in the workspace one thousand $(N=1,000) r_{i j n}$ range measurements were to be recorded by each of the four fixed beacons. The true distances $d_{i j}$ between each of the fixed beacons and mobile beaconantennas were to be computed using the measured coordinates. The errors $\varepsilon_{i j n}$ between the true distances and the measured rangeswere to be usedto estimate a non parametric model (frequency histogram) of the range error probability distribution $\mathrm{P}_{k}(\varepsilon)$ at each individual height.

\subsection{Range ERror Measurement in Foliage}

Depending onthe position of fruit in the canopy, a percentage of the LOS between a trailer beacon and the glove beacon will contain vegetation (leaves, branches, and fruits). This percentage may vary 
from $0 \%$ for fruits in the periphery of the canopy, to $100 \%$ for cases when the entire tree's half-

304

305

306

307

308

409

410

411

408

409

410

411

412

413

414

403

volume is between the glove and a trailer antenna, and when branches extend out to that trailer beacon antenna. The actual frequency with which each ratio is encountered depends on factors such as tree type, training system, pruning, and inter-row distance in the orchard. To estimate the range errors in the presence of foliage, equal numbers of range measurements were recorded for three percentages of vegetation coverage at approximately $35 \%, 70 \%$ and $100 \%$, at different heights. The true locations were measured with the Total Station. At each height, the errors from the three vegetation ratios were used to estimate the corresponding range error histograms. The tree used was a Bartlett pear tree (Pyrus communis 'Williams pear') of the open-vase architecture.

For each vegetation percentile data set, a beacon antenna (referred to as "fixed") was placed and held fixed at two different locations (No.1 and No.2 in Figure 10), each time atfivedifferent heights $(0.91,1.83,2.74,3.66$ and $4.5 \mathrm{~m})$. For each combination of location and height of the fixed antenna,a"mobile" beacon antenna was movedto ninedifferent locations on the other side of the tree, as shown in Figure 10, at a height of $2.32 \mathrm{~m}$ from the ground. This height is the same as the trailer's three beacons. Hence, 18 experiments were conducted for each height of the fixed beacon, and a total of 90 measurement sets were taken.

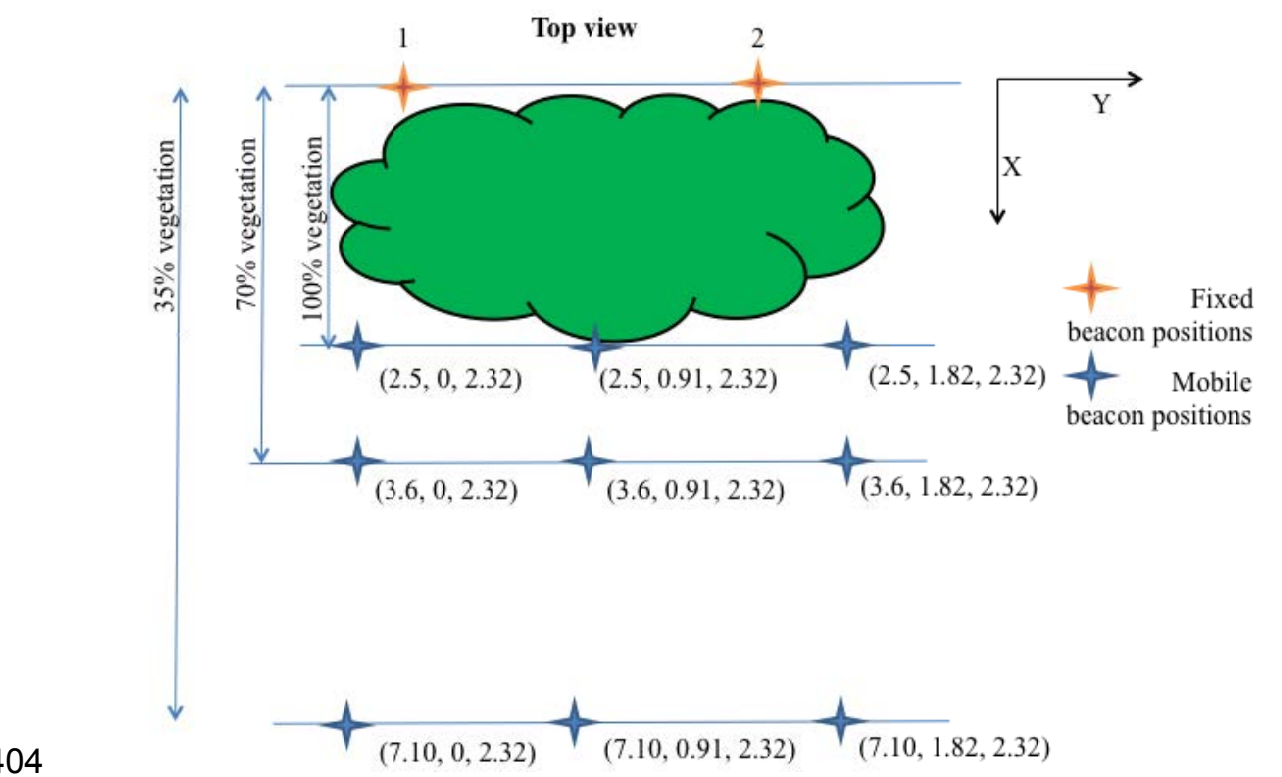


406

407

408

409

410

411

412

413

414

415

416

417

418

419

420

421

422

423

\section{EXPERIMENTAL RESULTS AND DISCUSSION}

All transmitter beacons were calibrated before the experiment to compensate for antenna cable length delays. The calibration was performed according to the manufacturer's suggested procedure.

\subsection{RANGe ERror Statistics In OPEn SPaCe}

The range error histograms that approximate distributions $\mathrm{P}_{k}(\varepsilon)$ were computed for five different heights $(\mathrm{k}=1,2, \ldots, 5)$, from 70,626 measurements at each height. Two-sample Kolmogorov-Smirnov tests were used for each possible pair of distributions $\mathrm{P}_{m}(\varepsilon), \mathrm{P}_{n}(\varepsilon)$, with $m, n \in\{1,2, \ldots 5\}$ and verified at the 5\% significance level that error data at different heights came from different distributions. The histograms for the lowest plane $\left(\mathrm{P}_{1}(\varepsilon)\right)$ and highest plane $\left(\mathrm{P}_{5}(\varepsilon)\right)$ are shown in Figure 11.
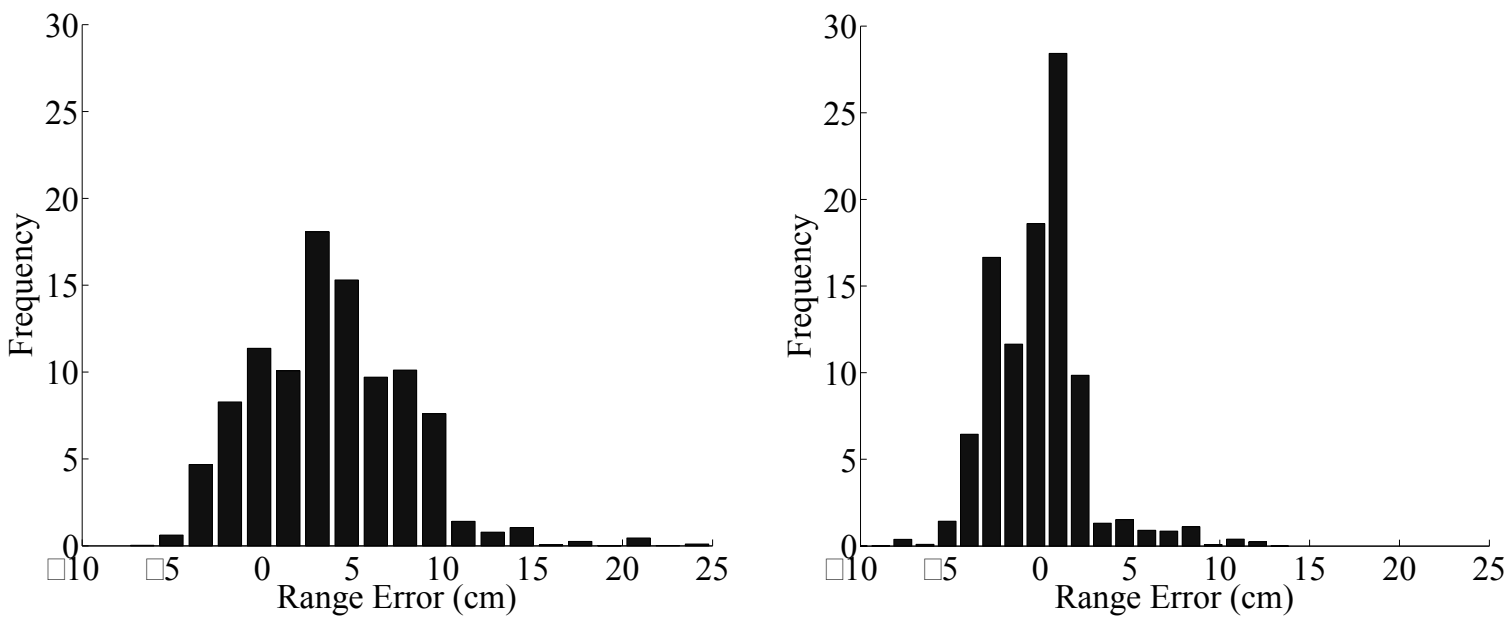

Figure 11: Open-space range error histograms at two heights: $P_{1}(\varepsilon)$ at $0.9 \mathrm{~m}$ (left) and $P_{5}(\varepsilon)$ at $4.5 \mathrm{~m}$ (right).

Error metrics of the distributions are given in Table 1 for each height and for the entire measurement volume (last column). Although all beacons had been calibrated before the experiments, the range error distributions were slightly biased. In theory, bias for UWB ranging systems should always be positive, because it results from delayed signal arrivals due to wave reflections. A possible reason for negative range errors and bias is electromagnetic field saturation. When the antennas are 
424 too close together, the returning signalmay enter a trailer RTRU directly through its antenna

425 connector, instead of entering from the antenna and propagating through the antenna cable.

426

427

428

\begin{tabular}{|lcccccc|}
\hline & $\mathrm{P}_{1}(\varepsilon)$ & $\mathrm{P}_{2}(\varepsilon)$ & $\mathrm{P}_{3}(\varepsilon)$ & $\mathrm{P}_{4}(\varepsilon)$ & $\mathrm{P}_{5}(\varepsilon)$ & ALL \\
Mean & 3.83 & 1.70 & 2.81 & 2.04 & -0.13 & 2.05 \\
Std. dev. & 4.22 & 3.89 & 3.90 & 2.98 & 2.57 & 3.80 \\
Mean absolute & 4.56 & 3.23 & 3.62 & 2.81 & 1.88 & 3.22 \\
$\begin{array}{l}\text { Root Mean Square } \\
\text { (RMS) }\end{array}$ & 5.7 & 4.24 & 4.81 & 3.61 & 2.58 & 4.32 \\
R90 & 9.2 & 6.88 & 8.37 & 5.98 & 3.86 & 7.48 \\
\hline
\end{tabular}

Table 1.Range error metrics in open space when LOS is unobstructed; they were calculated from experimentally derived error distributions $\mathrm{P}_{k}(\varepsilon)$ at five different heights (units in $\mathrm{cm}$ ).
429

430

431

432

433

434

435

436

437

438

439

440

441

442

The general trend was for range errors to decrease as height increased. At $2.7 \mathrm{~m}(k=3)$, the error seems to deviate from the trend. However, at a later point, it was noticed that a horizontal aluminum tube that provided structural support to the beacon poles was close to that height. Therefore, it is possible that it interfered in the signal propagation. This "anomaly" manifested itself in the trilateration error, too (see Figure 15 later).

\subsection{RANGe ErRor Statistics IN Foliage}

The error measurements were taken in a pear orchard at the UC Davis campus. Visual inspection indicated that the foliage density was typical of commercial orchard pear trees, similar to the ones visited for our data collection (described later). Unfortunately, these trees had been hedged, and their height did not exceed $4 \mathrm{~m}$. Therefore, the range error histograms that approximate distributions $\mathrm{P}_{k}(\varepsilon)$ were computed for four - instead of five - different heights $(k=1,2, \ldots, 4)$. A total of 18,000 measurements were taken at each height. Two-sample Kolmogorov-Smirnov tests were used for each possible pair of distributions $\mathrm{P}_{m}(\varepsilon), \mathrm{P}_{n}(\varepsilon)$, with $m, n \in\{1,2,3,4\}$, and verified at the $5 \%$ significance 
443 level that error data at different heights came from different distributions. The histograms for the 444 lowest plane $\left(\mathrm{P}_{1}(\varepsilon)\right)$ and highest plane $\left(\mathrm{P}_{4}(\varepsilon)\right)$ are shown in Figure 12.
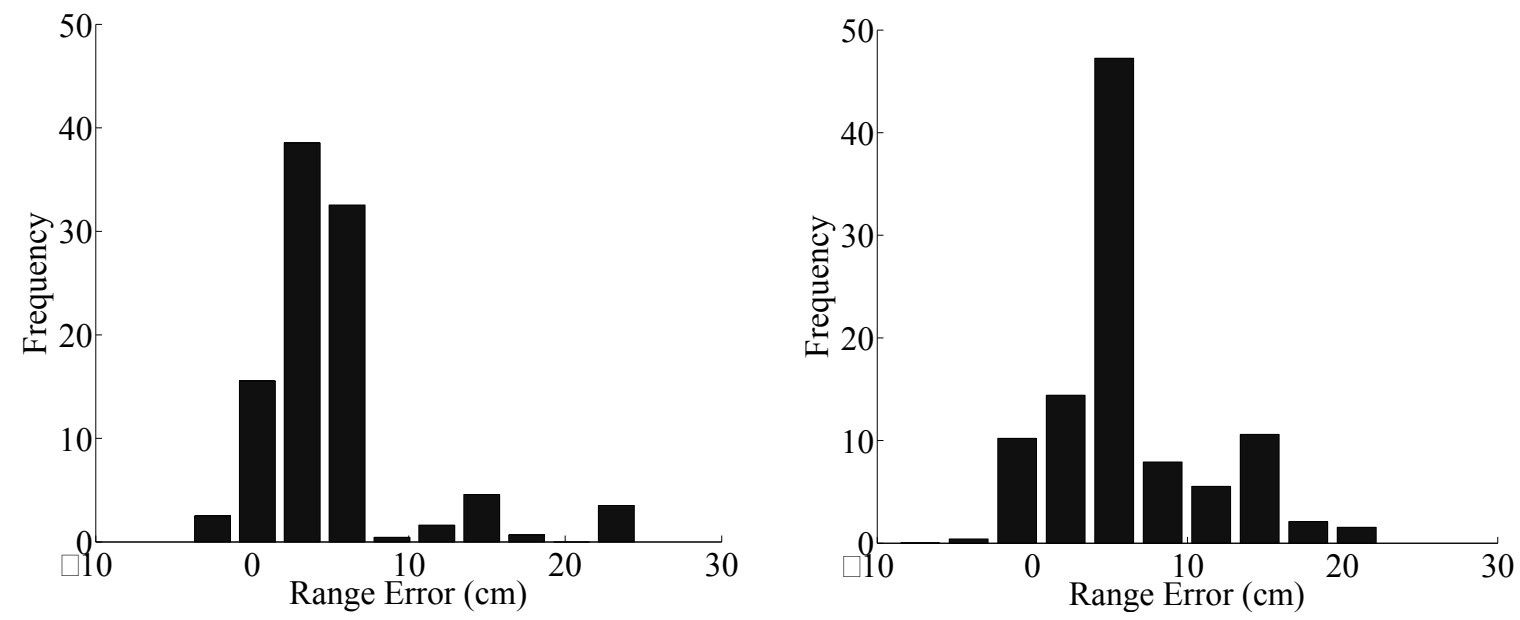

445

446

447

448

449

450

451

\begin{tabular}{|l|c|c|c|c|c|c|}
\hline & $\mathrm{P}_{1}(\varepsilon)$ & $\mathrm{P}_{2}(\varepsilon)$ & $\mathrm{P}_{3}(\varepsilon)$ & $\mathrm{P}_{4}(\varepsilon)$ & $\mathrm{P}_{5}(\varepsilon)$ & ALL \\
\hline Mean $(\bar{\varepsilon})$ & 5.16 & 4.52 & 6.34 & 6.39 & - & 5.60 \\
\hline Std. dev. & 4.93 & 2.56 & 6.11 & 5.05 & - & 4.90 \\
\hline Mean absolute & 5.28 & 4.63 & 6.96 & 6.63 & - & 5.88 \\
\hline $\begin{array}{l}\text { Root Mean Square } \\
\text { (RMS) }\end{array}$ & 7.13 & 5.20 & 8.81 & 8.14 & - & 7.45 \\
\hline R90 & 10.74 & 8.17 & 12.98 & 14.90 & - & 12.19 \\
\hline
\end{tabular}

452

A comparison of Tables 1 and 2 reveals that, on the whole, the presence of foliage increased the mean error (bias) by $173 \%$, the standard deviation by $28.9 \%$, the mean absolute error by $82.6 \%$, the 455

Figure 12: Range error histograms in foliage at two heights: $P_{1}(\varepsilon)$ at $0.9 \mathrm{~m}$ (left) and $P_{4}(\varepsilon)$ at $3.6 \mathrm{~m}$ (right).

Table 2.Range error metrics when LOS contains vegetation; they were calculated from experimentally derived error distributions $\mathrm{P}_{k}(\varepsilon)$ at four different heights(units in $\mathrm{cm}$ ).

In Table 2, error metrics of the distributions are given both for each height and for the entire measurement volume (last column). 
457 upper parts of the open-vase Bartlett pear tree that was used for testing. In general, lower limbs on 458 such trees tend to lose vigor, and upper limbs tend to grow above lower ones and shade them out 459 (Mitcham and Elkins, 2007). More data would be needed to support this hypothesis. The histograms of 460 all range error measurements in open space and foliage are juxtaposed in Figure 13.

461
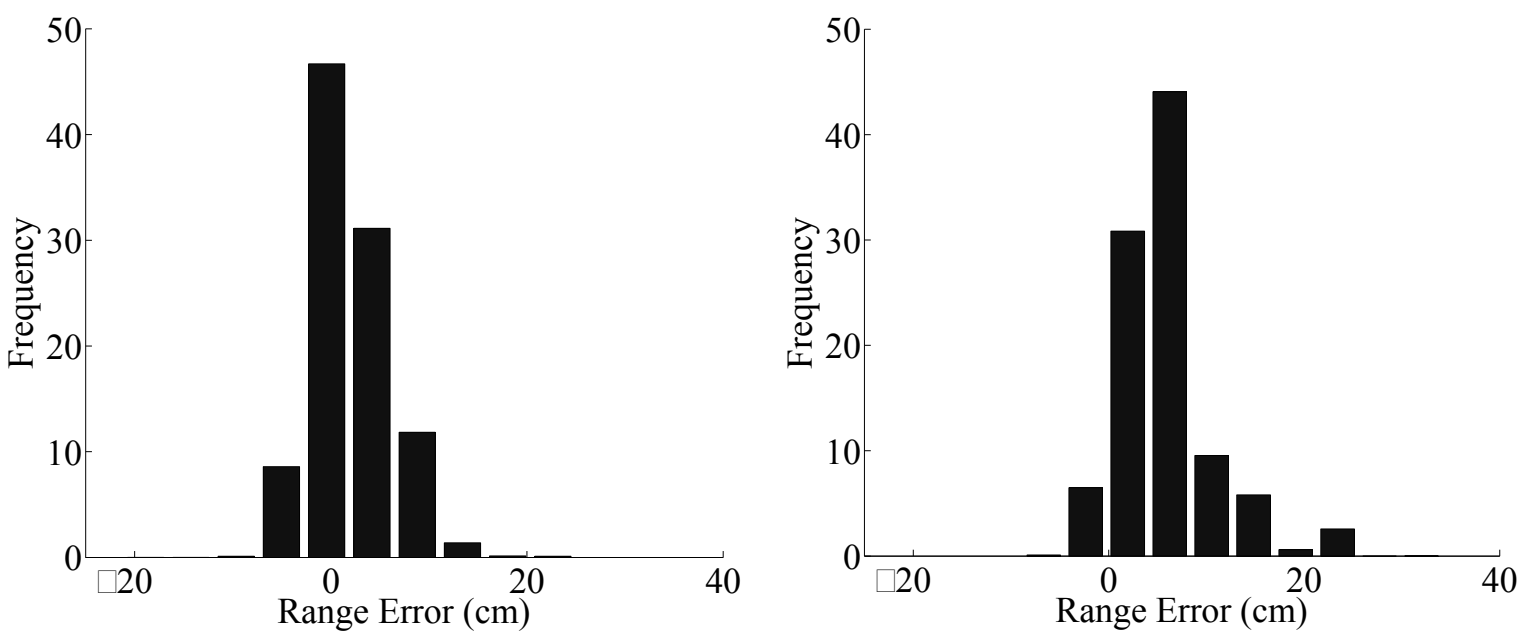

462

463

464

465

466

467

468

469

470
Figure 13: Range error histograms of all sampled points inside the system's measurement volume in open space (left) and with vegetation (right).

The presence of foliage has "shifted" the histogram to the right resulting in larger bias, and has also increased the standard deviationto $4.9 \mathrm{~cm}$, compared to $3.8 \mathrm{~cm}$ in open space.

\subsection{TRILATERATION ERRORS IN OPEN SPACE - VALIDATION}

The open-space trilateration errors were measured, and their spatial distribution is shown in Figure 14. 


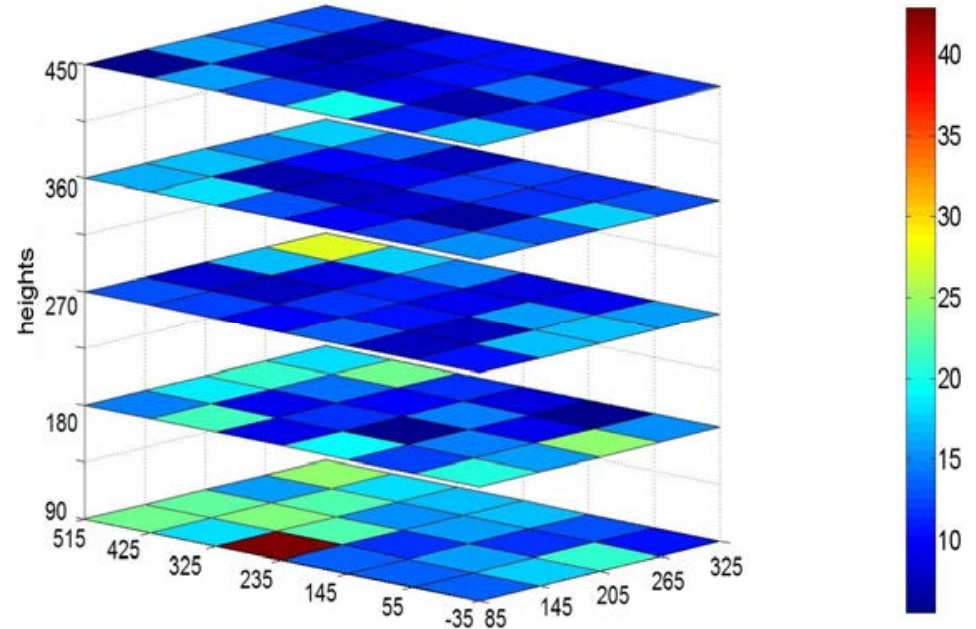

trailer length

into the canopy

Figure 14: Spatial distribution of measured trilateration DRMS error at five heights inside the system's measurement volume.

Metrics of themeasured trilateration error at different heights and over the entire measurement volume(last row labeled "Overall”) are given in Table 3. The standard deviations reflect the precision of the measurements in the three axes.

Table 3. Open-space trilateration accuracy metrics calculated from measurements of uniformly sampled points at five different heights inside the system's measurement volume (units in $\mathrm{cm}$ ).

\begin{tabular}{|cccccc|}
\hline & DRMS & ABSEx \pm std & ABSEy \pm std & ABSEz \pm std & R90 \\
Height 1 & 12.9 & $7.2 \pm 6.1$ & $3.1 \pm 2.3$ & $6.3 \pm 4.7$ & 16.9 \\
Height 2 & 11.6 & $5.2 \pm 4.9$ & $4.2 \pm 2.9$ & $6.0 \pm 4.5$ & 17.1 \\
Height 3 & 9.8 & $3.4 \pm 2.9$ & $5.1 \pm 3.4$ & $4.9 \pm 3.8$ & 13.9 \\
Height 4 & 8.9 & $4.0 \pm 3.7$ & $3.5 \pm 2.1$ & $4.5 \pm 3.5$ & 13.5 \\
Height 5 & 7.7 & $4.3 \pm 3.2$ & $2.6 \pm 2.1$ & $3.6 \pm 2.5$ & 11.4 \\
Overall & 10.3 & $4.8 \pm 4.5$ & $3.7 \pm 2.8$ & $5.1 \pm 4.0$ & 15.1 \\
\hline
\end{tabular}

The general trend was that trilateration errors decreased as height increased. This is in agreement with previous results for UWB-based trilateration close to the ground (Saidiet al., 2011). The effect 

approximately $60 \%$ of the corresponding values at $0.9 \mathrm{~m}$. Also, the errors along the $\mathrm{X}$ and Z-axes were significantly larger than the Y-axis errors at all heights, except for $2.7 \mathrm{~m}$ (Height 3). The explanation for this lies in the geometrical configuration of the trailer beacons. It is known 488 (Manolakis, 1996) that, the bigger the distance between beacons along an axis, the better the 489 localization accuracy along this axis. The trailer beacons were farther apart in the Y-axis than in the X 490 or Z-axes.

491 The Monte Carlo simulation was implemented in Matlab, and was executed using the open-space 492 range error histograms that resulted from our measurements. The metricsof the simulated errors are 493 reported in Table 4. For comparison purposes, the DRMS errors from measurements and Monte Carlo 494 simulation are presented in Figure 15.

495 Table 4. Open-space trilateration accuracy metrics calculated via Monte-Carlo simulation at 496 uniformly sampled points, at five different heights inside the system's measurement volume (units in $497 \mathrm{~cm})$.

\begin{tabular}{|cccccc|}
\hline & DRMS & ABSEx \pm std & ABSEy \pm std & ABSEz \pm std & R90 \\
Height 1 & 13.7 & $9.4 \pm 7.3$ & $3.7 \pm 2.9$ & $4.0 \pm 2.7$ & 20.7 \\
Height 2 & 11.7 & $8.3 \pm 6.5$ & $3.0 \pm 2.4$ & $2.5 \pm 2.0$ & 18.2 \\
Height 3 & 11.7 & $8.1 \pm 6.2$ & $3.2 \pm 2.5$ & $3.2 \pm 2.3$ & 18.0 \\
Height 4 & 8.7 & $6.0 \pm 4.7$ & $2.4 \pm 1.9$ & $2.4 \pm 1.7$ & 13.4 \\
Height 5 & 6.9 & $4.8 \pm 4.0$ & $1.8 \pm 1.5$ & $1.4 \pm 1.2$ & 11.0 \\
Overall & 10.8 & $7.4 \pm 6.1$ & $2.8 \pm 2.4$ & $2.7 \pm 2.2$ & 16.9 \\
\hline
\end{tabular}


499

500

501

502

503

504

505

506

507

508

509

510

511

512

513

514

515

516

517

518

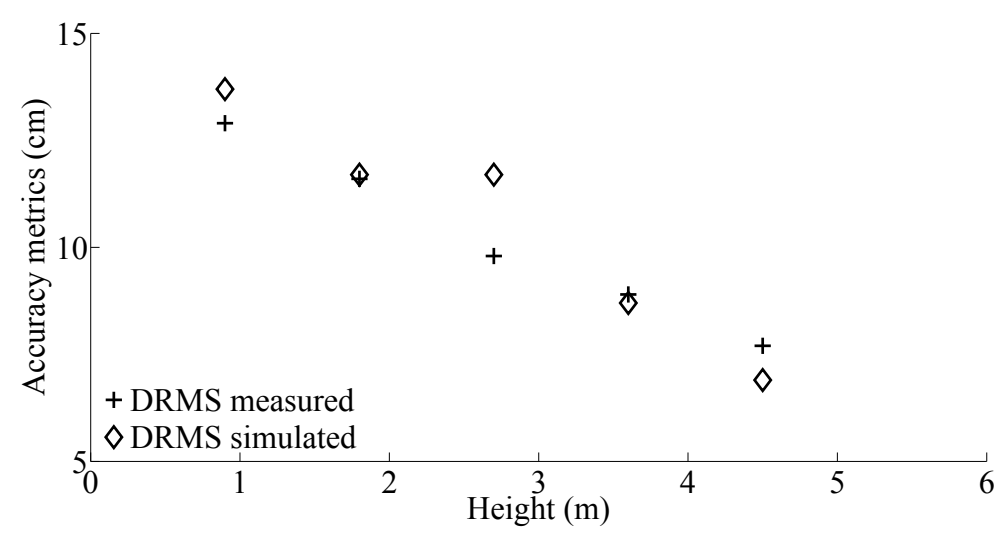

Figure 15: Measured and simulated/predicted trilateration DRMS error at all sampled points in the measurement volume.

The graph shows that the simulator captured the general trend of decreasing trilateration errors with increasing height. From Tables 3 and 4, it can be deduced that, over the entire measurement volume, the simulator overestimated ABSEx by $54.2 \%$, underestimated ABSEy by $24.3 \%$, and underestimated ABSEz by $47.1 \%$. However, since non-directional range error statistics were used for the MonteCarlo error assessment method, non-directional trilateration error metrics are better suited to evaluate it. These metrics were much better: DRMS was overestimated by only $4.9 \%$, and R90 was overestimated by $11.9 \%$. Therefore, the Monte-Carlo method can be used for trilateration error assessment.

\subsection{Trilateration ERrors in Tree CanOpies - Simulation}

The range error distributions that were determined experimentally in foliage were used to execute the Monte Carlo simulation.1,000 range error samples were generated for each point of the grid at each height. The error statistics of the simulated errors are reported in Table 5.

Table 5.Estimated trilateration accuracy metrics in the presence of vegetation calculated via MonteCarlo simulation at uniformly sampled points, at five different heights inside the system's measurement volume (units in $\mathrm{cm}$ ).

DRMS $\quad$ ABSEx \pm std $\quad$ ABSEy \pm std $\quad$ ABSEz \pm std $\quad$ R90




\begin{tabular}{lccccc} 
Height 1 & 16.2 & $9.8 \pm 9.8$ & $4.0 \pm 3.9$ & $5.2 \pm 3.4$ & 27.8 \\
Height 2 & 9.4 & $5.7 \pm 4.4$ & $2.9 \pm 2.0$ & $4.6 \pm 1.8$ & 13.5 \\
Height 3 & 19.4 & $12.8 \pm 10.3$ & $5.3 \pm 4.3$ & $6.5 \pm 4.1$ & 30.1 \\
Height 4 & 16.2 & $10.3 \pm 8.1$ & $4.8 \pm 3.7$ & $6.5 \pm 3.5$ & 24.4 \\
Height 5 & - & - & - & - & - \\
Overall & 15.7 & $9.6 \pm 8.8$ & $4.3 \pm 3.7$ & $5.7 \pm 3.4$ & 24.9 \\
\hline
\end{tabular}

519

520

521

522

523

524

525

526

527

528

529

530

531

532

533

534

535

536

537

538

\subsection{ESTIMATION OF FRUIT LOCATIONS IN ORCHARDS}

Fruit positions were measured using the developed system in eight (8) commercial orchards in California, in July and August 2014. The primary goal was to assess the system's fruit position digitization rates in different orchards under typical harvesting operations. The digitization rates depend on non-picking (non-productive) times, which are mainly the result of using ladders. Therefore, trees were not randomized; instead, fruit positions from consecutive trees ina random row of each orchard were digitized.A total of 32,193 fruit positions were measured from 66 trees:24 clingpeach trees (Prunus persica(L.) Batsch) and 42 pear trees (Pyrus communis 'Williams pear'). Tree architectures were: Standard Open Vase (SOV); High-Density Open Vase (HDOV); Trellised (T); High Density Trellised (HDT). Tree information and average fruit position digitization rates are shown in Table 6.

Table 6. Fruit position measurement rates for several cling-peach and pear tree architectures.

\begin{tabular}{|l|c|c|c|c|c|}
\hline Canopy & Number of & Number & Tree height $(\mathrm{m})$ & Tree age & Digitizing rate \\
\hline
\end{tabular}




\begin{tabular}{|l|c|c|c|c|c|}
\hline architecture & trees & of fruits & & (years) & (Fruits / minute) \\
\hline SOV - Peach & 4 & 5142 & 3.7 & 12 & 14.13 \\
\hline SOV - Peach & 6 & 4010 & 4.1 & 22 & 11.2 \\
\hline SOV - Peach & 4 & 5075 & 4.9 & 14 & 11.71 \\
\hline HDOV - Peach & 10 & 3999 & 3.4 & 4 & 11.93 \\
\hline SOV - Pear & 3 & 3694 & 4.3 & 33 & 9.09 \\
\hline SOV - Pear & 4 & 3329 & 4.1 & 15 & 8.79 \\
\hline HDT - Pear & 28 & 4329 & 2.7 & 9 & 11.48 \\
\hline T - Pear & 7 & 2615 & 4.9 & 12 & 7.99 \\
\hline
\end{tabular}

539

540

541

542

543

544

545

546

547

548

549

550

551

552

553

554

555

556

557

558

The fruit position digitizing rates include the time required by pickers to locate, reach, pick, place, transport fruit to bins, and move ladders, as well as the time required by researchers to pull (manually) the data acquisition trailer along the orchard rows. The digitizing rates for cling peach and pear trees varied from 11 to 14 , and from 8 to 11.5 fruits per minute, respectively. In particular, for SOV peach trees, the digitizing rate was 12.3 fruits per minute based on 14,227 fruits collected from 14 trees $(1.4$ hours per tree), while for SOV pear trees it was 8.9 fruits per minutebased on 7,023 fruits collected from 7 trees (1.9 hours per tree). The main reason behind this difference is that SOV pear trees have a more complex canopy structure than cling peach trees of the same architecture; therefore, pickers needed more time to move ladders and position themselves on them. The digitization rate as a function of total number of harvested fruits - regardless of fruit type and tree architecture - is shown in Figure 16. There seems to exist a linear correlation between digitization ratesand total number of harvested fruits (Pearson's $r=0.9$ ). If the same number of fruits were located sparsely on large trees rather than closer together on small trees, the corresponding digitization rate would be expected to be lower. To capture this, spatial density of harvested fruits (fruits $/ \mathrm{m}^{3}$ ) was used as an independent variable, and its relationship to harvesting rate is shown in Figure 17. Spatial density was calculated by dividing the total number of harvested fruits over the volume occupied by all trees, including the space between trees in the row. A linear correlation seems to exist between fruit spatial density and digitization rate (Pearson's $r=0.7$ ), which becomes mush stronger (Pearson's $r=0.9$ ) if the first data point is ignored (at 3.7 fruits $/ \mathrm{m}^{3}, 11.48$ fruits $/ \mathrm{min}$ ). This point corresponds to high-density pear trees 
565 (HDT - Pear) with low height at $2.7 \mathrm{~m}$, which require significantly less usage of ladders for

566 harvesting. The correlation can be explained by the fact that when many fruits are harvested in smaller

567 volume, picking (i.e. digitizing) time dominates over non-productive, i.e. non-picking time.

564

565

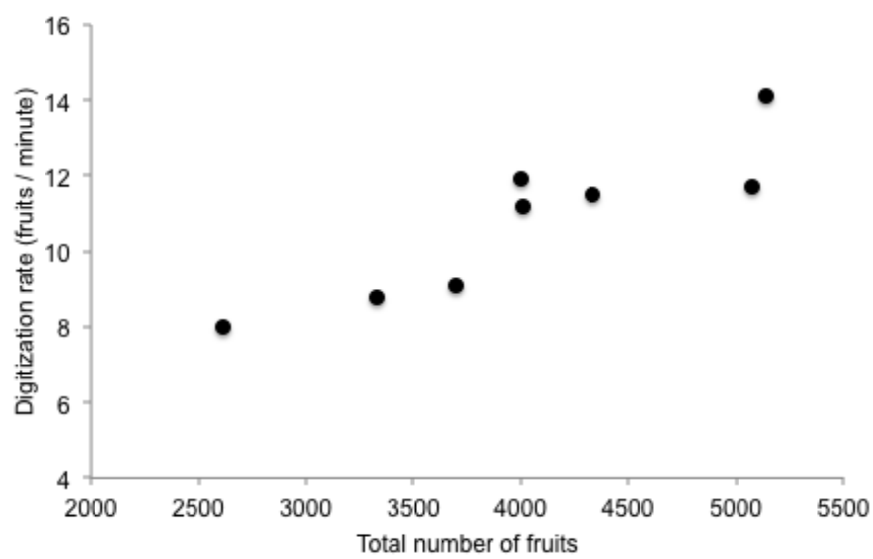

Figure 16. System's fruit position measurement rate versus total number of harvested fruits.

566

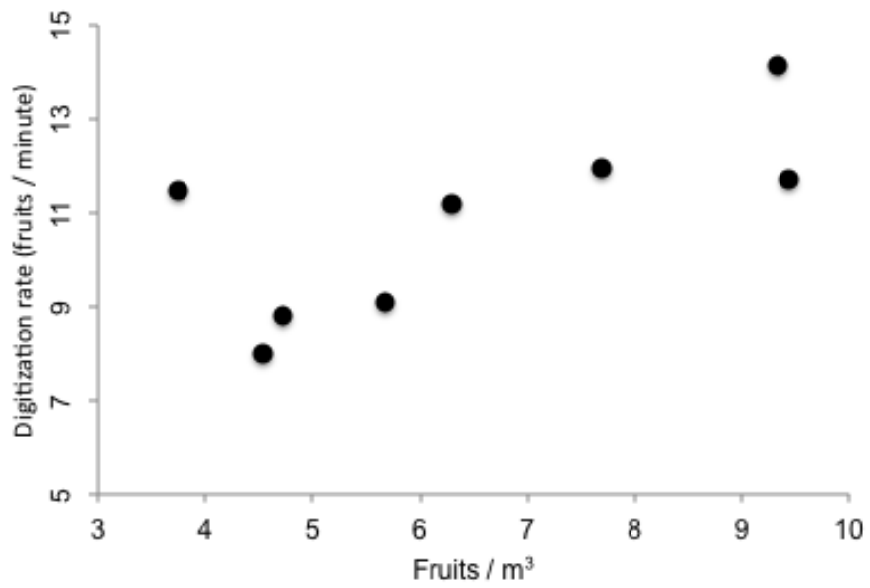

Figure 17. System's fruit position measurement rate versus spatial density of harvested fruits (fruits $/ \mathrm{m}^{3}$ ).

Next, a fruit position 3D map from one field experiment in a pear orchard in Lakeport, CA is 577 presented along with some spatial statistics. The Bartlett pear trees were approximately $5 \mathrm{~m}$ tall. The 578 data were collected from seven trees along a row; an aerial view of the part of the orchard row is 579 shown inFigure 18. 


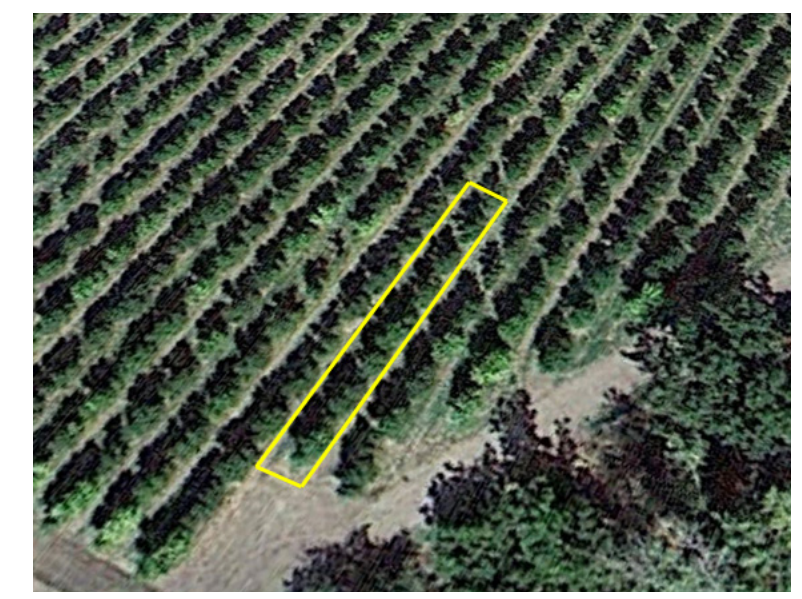

Figure 18: Satellite image of Bartlett pear orchard. Fruit positions were measured from the seven trees inside the yellow rectangle.

A map of the fruit locations in $3 \mathrm{D}$ is shown in Figure 19. The horizontal fruit coordinates are georeferenced in UTM, but, for the sake of readability, they are expressed in the figure with respect to the trailer's initial position. Furthermore, heights are with respect to the ground $(0 \mathrm{~m})$. Maps like these could be used by growers to assess how their flower and fruit thinning strategies affect fruit distribution, with the purpose ofadjusting them accordingly.

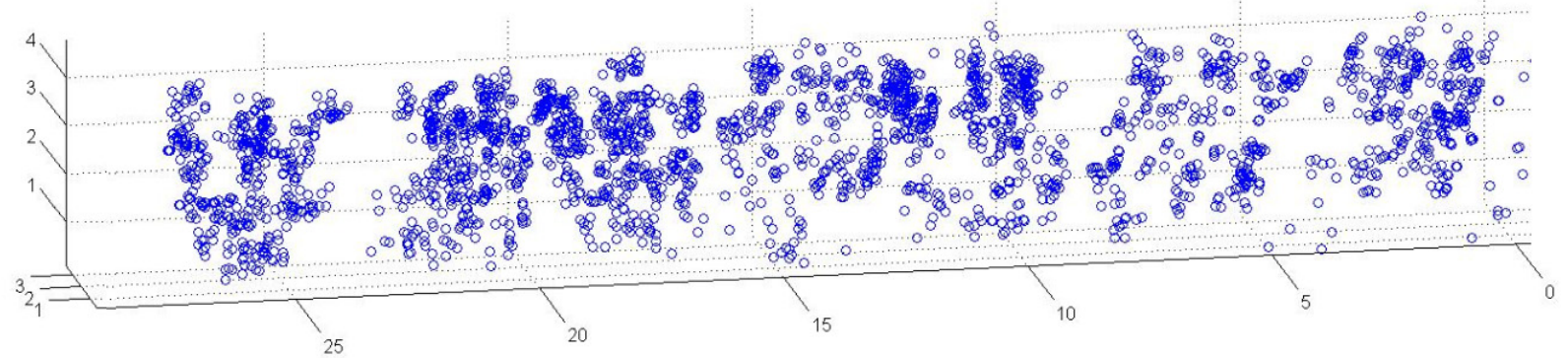

Figure 19: 3D pear-fruit locations measured from seven trees in a row. Coordinates $(\mathrm{m})$ are expressed with respect to the trailer's initial position and to the ground $(0 \mathrm{~m})$.

579

The yield of each tree can be seen in Figure 20.Although the trees were next to each other in the same row and had the same age, yield variability was significant: minimum and maximum yields were 301 and 429 respectively (42.6\% difference). The mean was 373.6 fruits, while the standard deviation 43.4 fruits. Such information - if it becomes available for more trees - could be used by farmers to identify problems--such as water and nutrient availability--and manage the orchard more precisely. 


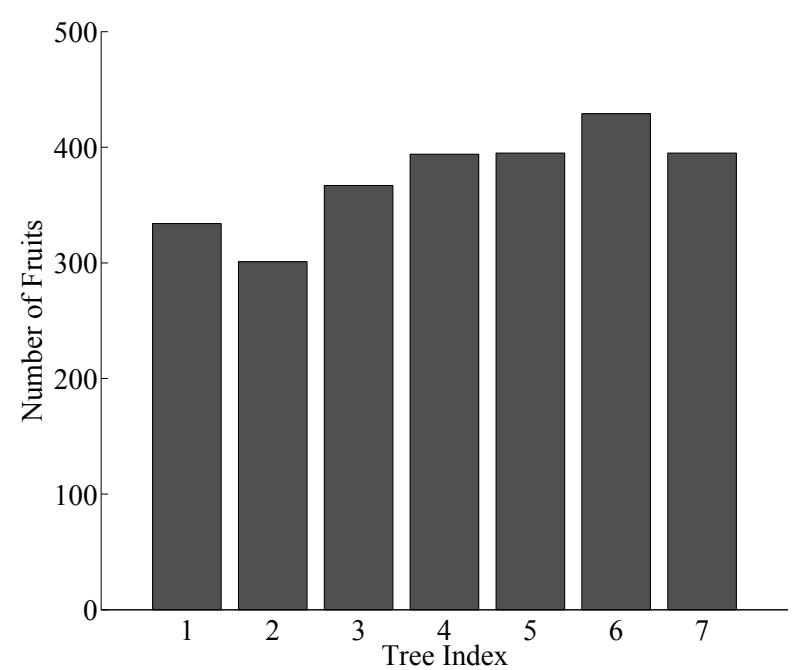

Figure 20: Bar chart showing the total number of fruits (yield) on each tree.

587

588

589

590

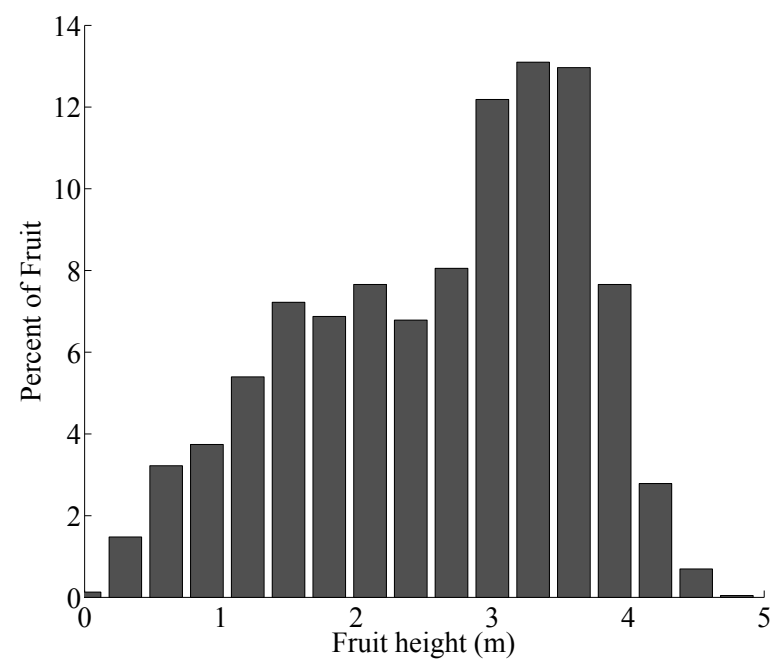

Figure 21: Bar chart depicting the normalized histogram of fruit heights ( $\mathrm{z}$ coordinates). $1 \mathrm{~m}$.

593 bear fruit from near the ground to their tops. Approximately $60 \%$ of the fruit is above $2.2 \mathrm{~m}$; i.e., the

594 average picker on the ground cannot reach it.Such information can be very useful for the design and

595 fine-tuning of harvesting and harvest-aiding equipment, such as orchard platforms. 


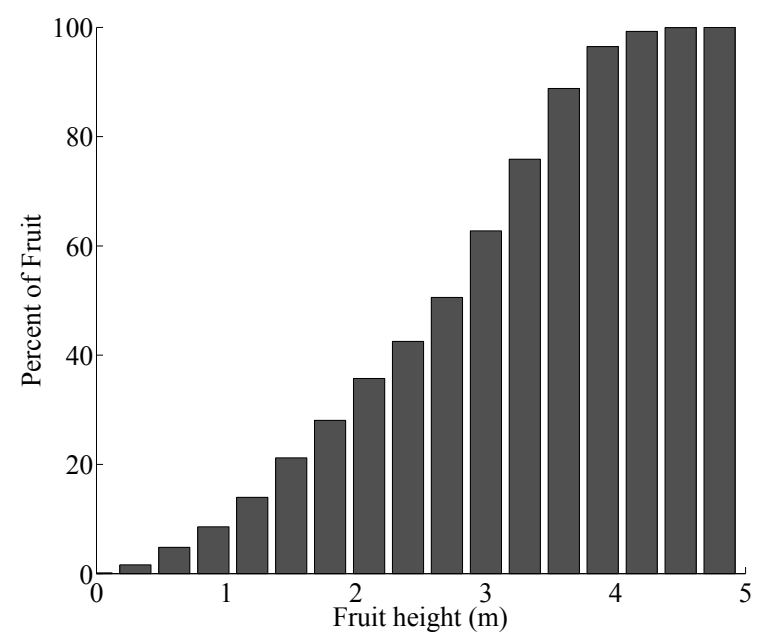

Figure 22: Bar chart depicting the cumulative histogram of fruit heights ( $\mathrm{z}$ coordinates).

The normalized histogram of the fruit distances from the row-centers is shown in Figure 23. The

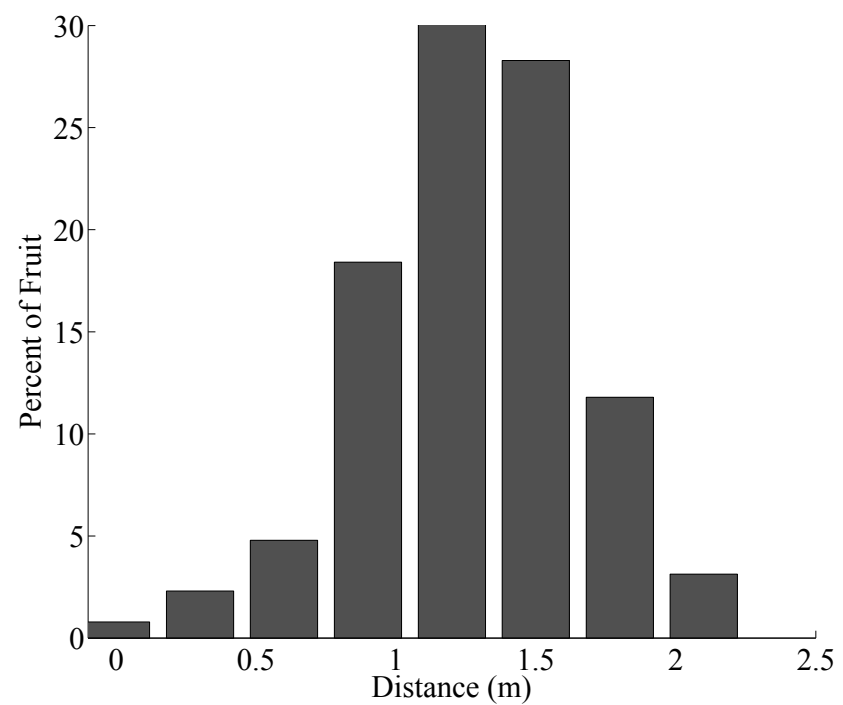

Figure 23: Normalized histogram of fruit horizontal minimum distance from the left and right row centers.

\section{Conclusions}

A novel approach to fruit localization, which utilizes high-frequency radio signals and trilateration 

than existing reported methods. As a consequence, it renders the system practical for real-world use.

612 In open space, the RMS error between the estimated and true distance (DRMS) in the system's measurement volume was measured to be $10.3 \mathrm{~cm}$. The error's $90^{\text {th }}$ percentile $(\mathrm{R} 90)$ was $13.1 \mathrm{~cm}$. The 614 corresponding errors for locations in tree canopies had to be estimated using Monte Carlo 615 simulation, and were calculated to be equal to $15.7 \mathrm{~cm}$ and $24.9 \mathrm{~cm}$ respectively. This accuracy is 616 about an order of magnitude worse than that of commercial magnetic field based digitizers, which can 617 work without LOS (unlike surveying instruments). However, the range of these systems is limited to 1 $618-1.5 \mathrm{~m}$, whereas the developed system can handle very large trees, up to $5 \mathrm{~m}$ tall.

619 In relative terms, the worst-case position accuracy along each axis can be calculated by dividing the 620 corresponding error(ABSEx, ABSEy, or ABSEz) by the distance between the fruits that are as far away as possible from each other along the corresponding axis. Distances along $\mathrm{x}, \mathrm{y}$ and $\mathrm{z}$ are $3.49 \mathrm{~m}$, $2.7 \mathrm{~m}, 1.67 \mathrm{~m}$, respectively. The relative fruit position accuracies in the $\mathrm{x}, \mathrm{y}$ and $\mathrm{z}$-axes were calculated to be $2.7 \%, 1.6 \%$ and $3.4 \%$, respectively. If such fruit position data are used as input for 624 fruit reachability and picking cycle simulations, the computed metrics will also contain uncertainty. 625 The effects of such fruit position uncertainties on the value of the computed metrics for design 626 purposes cannot be quantified at this point. However, two important facts should be considered. First, 627 since standard deviations are known, uncertainty in fruit positioning can be propagated via the 628 harvesting simulator, and translated into variance of harvesting metrics. Consequently, quantitative 629 estimates of the effects of fruit position uncertainty on harvesting metric uncertainty can be computed 630 and evaluated. Second, it is the difference in the calculated performance metrics between alternative 631 designs that drives the model-based design process, and not the values of the metrics themselves. This 632 implies that uncertainty/variance in a harvesting metric may not be important, as long as the expected 633 values of the metrics of two alternative designs are far apart from each other. Therefore, the relatively 634 small fruit position measurement uncertainties may constrain the resolution of the design space being 
explored —in other words, the length increment of a robot arm link in a parametric optimization—but they are not expected to undermine the validity of model-based design for robotic harvesters.

\section{ACKNOWLEDGEMENTS}

We would like to thank Rachel Elkins (University of California Cooperative Extension, Lake and Mendocino Counties), Janine Hasey (University of California Cooperative Extension Farm Advisor, Sutter \& Yuba Counties), Roger Duncan (UC Extension Pomology \& Viticulture Farm Advisor, Stanislaus County), and Chuck Ingels (University of California Cooperative Extension, Sacramento County) for their invaluable help in organizing the experiments. We would also like to thank numerous California growers for letting us gather data during the harvesting season and acknowledge the contributions of students Jason Wong and Garren Lewis for their help during the experiments. Finally, we would like to thank the anonymous reviewers for their constructive comments. This research was funded by the California Pear Advisory Board, the Cling Peach Mechanization Research Fund, and USDA-NIFA AFRI Competitive Grant 2016-67021-24532.

\section{REFERENCES}

Chehri, A., Fortier, P., and Tardif, P. M. (2009). UWB-based sensor networks for localization in mining environments. Ad Hoc Networks. 7(5): 987-1000.

Cheng, T., Venugopal, M., Teizer, J., and Vela, P. A. 2011. Performance evaluation of ultra wideband technology for construction resource location tracking in harsh environments. Automation in Construction. 20(8): 1173-1184.

Cox, M.G., Harris, P.M., Siebert B.R.-L. 2003. Evaluation ofmeasurement uncertainty based on the propagation of distributionsusing Monte Carlo simulation. Measurement Techniques46 (9), 824-833.

Edan, Y., T. Flash,U.M.Peiper, I. Shmulevich, and Y. Sarig. 1991. Near-minimum-time task planning for fruit-picking robots. IEEE Transactions on Robotics and Automation, 7(1): 48-56.

Harrell, R. Economic analysis of robotic citrus harvesting in Florida. Transactions of the ASAE, 30 (2): 
JCGM 101:2008, (2008). Evaluation of measurement data - Guide to the expression of uncertainty in measurement. Propagationof distributions using Monte Carlo method, Joint Committeefor Guides in Metrology, First edition.

664 665

666

667

668

669

670

671

672

673

674

675

676

677

678

679

680

681

682

683

684
Juste, F., C.Gracia, E. Molto, R.Ibanez, and S. Castillo. 1988. Fruit bearing zones and physical properties of citrus for mechanical harvesting. R. Goren and K. Mendel (Eds.), Proceedings of the 6th International Citrus Congress, Tel Aviv, Israel: Balaban Publishers, pp. 1801-1809.

Lee, B.S., and U.A. Rosa. 2006. Development of a canopy volume reduction technique for easy assessment and harvesting of Valencia citrus fruits. Transactions of the ASABE, 49(6): 1695-1703

Manolakis, D.E. 1996. Efficient solution and performance analysis of 3-D position estimation by trilateration. IEEE Transactions on Aerospace Electronic Systems, 32(4), 1239-1248.

Mitcham, E. J., Elkins, R. B. 2007. Pear Production and Handling Manual, Chapter 11, pp. 77. University of California, Agriculture and Natural Resources, Communication Services. Publication 3483, Oakland, CA, USA.

Navidi, W., Murphy Jr. W. S., Hereman, W. 1998. Statistical methods in surveying by trilateration,” Comput. Stat. Data Anal. 27, 209-217.

Sahinoglu, Z., Gezici, S. and Guvenc, I. 2008. Ultra-wideband positioning systems. Cambridge, New York.

Saidi, K. S., Teizer, J., Franaszek, M., \& Lytle, A. M. 2011. Static and dynamic performance evaluation of a commercially-available ultra wideband tracking system. Automation in Construction, 20(5), 519530.

Sangodoyin, S., Niranjayan, S., Molisch, A.F. 2013. A Measurement-Based Model for Outdoor NearGround Ultrawideband Channels. IEEE Transactions on Antennas and Propagation, 64(2): 740-751.

Schertz, C. E., and G. K. Brown. 1966. Determining Fruit-Bearing Zones in Citrus. Transactions of the ASAE, 9(3): 366-368. 
Sinoquet, H., P. Rivet, and C. Godin. 1997. Assessment of the three-dimensional architecture of walnut trees using digitising. Silva Fennica 31(3): 265-273.

Sivaraman, B., and T.F. Burks. 2006. Geometric performance indices for analysis and synthesis of manipulators for robotic harvesting. Transactions of the ASABE, 49(5): 1589-1597.

Sivaraman, B., and T.F. Burks. 2007. Robot Manipulator for Citrus Harvesting: Configuration Selection. ASABE Paper No. 071144. St. Joseph, Mich.: ASABE.

Smith, G.S., J.P. Curtis, and C.M. Edwards. 1992. A Method for Analysing Plant Architecture as it Relates to Fruit Quality Using Three-dimensional Computer Graphics. Annals of Botany, 70(3): 265269.

Smith, G.S., and J.P. Curtis. 1996. A fast and effective method of measuring tree structure in 3 dimensions. R. Habib and Ph. Blaise (Eds.), Proceedings of the 4rth International Symposium on Computer Models on Fruit Research, Acta Hort. (ISHS) 416:15-20.

Takahashi, T., S. Zhang, and H. Fukuchi. 2002. Measurement of 3-D locations of fruit by binocular stereo vision for apple harvesting in an orchard. ASABE Paper No. 021102. St. Joseph, Mich.:ASABE.

Van Henten, E.J., Van't Slot, D.A., Hol, C.W.J., Van Willigenburg, L.G. 2009. Optimal manipulator design for a cucumber harvesting robot. Computers and Electronics in Agriculture, 65(2):247-257.

Vougioukas, S., Anastassiu, H.T., Regen, C., Zude, M. (2013). Influence of foliage on radio path losses (PLs) for wireless sensor network (WSN) planning in orchards. Biosystems Engineering, 114(4), 454465.

Wang, Q., Nuske, S., Bergerman, M. and Singh, S. (2013). Automated crop yield estimation for apple orchards. Experimental Robotics, pp. 745-758. Springer International Publishing.

\section{APPENDIX: GEO-REFERENCING OF THE TRAILER BEACON ANTENNAS}

Our goal is to calculate the instantaneous georeferenced 3D position of each $j$ th beacon antenna expressed in world frame coordinates $\left(b x_{j}, b y_{j}, b z_{j}\right)$. The reason is that, given these four positions and 
710

711

712

713

714

715

716

717 the trailer beacons, ${ }^{T F} \mathbf{b}_{j}=\left[b x_{j} b y_{j} b z_{j} 1\right]^{T}, j=1,2,3,4 ;$ Rover GPS receiver origin, 718

the four distances between the beacon antennas and a glove antenna, we can calculate the 3D position of the glove antenna using trilateration. Next, we show how to compute this.

First, the following reference frames need to be defined: world frame (WF); initial trailer frame $(I T F)$ at the beginning of data collection; initial attitude sensor frame (YTF) at the beginning of data collection; instantaneous trailer frame (TF); instantaneous Rover GPS frame (GPSF); instantaneous attitude sensor frame $(A F)$. Let us also define the following positions as homogeneous-coordinate vectors expressed with respect to the trailer frame: receive-transmit center points on the antennas of ${ }^{T F} \mathbf{p}_{\text {GPSF }}=\left[\Delta x_{g p s} \Delta y_{g p s} \Delta z_{g p s} 1\right]^{T} ;$ and the origin of attitude sensor: ${ }^{T F} \mathbf{p}_{Y F}=\left[\Delta x_{y e i} \Delta y_{y e i} \Delta z_{y e i} 1\right]^{T}$. All the above positions were measured manually, after the beacons, GPS, and attitude sensor were rigidly placed on the trailer.

Let the homogeneous coordinates of the $j$ th beacon antenna with respect to the world frame be ${ }^{W F} \mathbf{b}_{j}=\left[b x_{j} b y_{j} b z_{j} 1\right]^{T}$. The position of the origin of the trailer frame expressed in the Rover GPS frame is ${ }^{G P S F} \mathbf{p}_{T F}=\left[-\Delta x_{g p s}-\Delta y_{g p s}-\Delta z_{g p s} 1\right]^{T}$. Let us define a matrix $I$ as,

$$
I=\left(\begin{array}{lll}
1 & 0 & 0 \\
0 & 1 & 0 \\
0 & 0 & 1 \\
0 & 0 & 0
\end{array}\right)
$$

Then, the homogeneous $(4 \times 4)$ coordinate transformation matrix of the instantaneous Rover GPS frame GPSF with respect to the instantaneous trailer frame $T F$ is ${ }^{T F} H_{G P S F}=\left[{ }^{I}{ }^{T F} \mathbf{p}_{G P S F}\right]$, and the transformation matrix of TF with respect to GPSF is ${ }^{G P S F} H_{T F}={ }^{T F} H_{G P S F}^{-1}=\left[I^{G P S F} \mathbf{p}_{T F}\right]$. The instantaneous trailer position and orientation measurements are given next: the position of the trailer's Rover GPS is ${ }^{W F} \mathbf{p}_{\text {GPSF }}=\left[x_{\text {gps }} y_{\text {gps }} z_{g p s} 1\right]^{T} ;$ the rotation of the attitude sensor with respect to the initial attitude sensor frame $(Y T F)$ is: 


$$
{ }^{\mathrm{YTF}} R_{\mathrm{TF}}=\left[\begin{array}{ccc}
c \theta c \varphi & s \psi s \theta-c \psi c \theta s \varphi & c \psi s \theta+c \theta s \psi s \varphi \\
s \varphi & c \psi c \varphi & -c \varphi s \psi \\
-c \varphi s \theta & c \theta s \psi+c \psi s \theta s \varphi & c \psi c \theta-s \psi s \theta s \varphi
\end{array}\right]
$$

734

735 Since the GPS and attitude sensors are rigidly attached to the trailer, the following transformations 736 are equal, i.e., ${ }^{Y T F} R_{A F}={ }^{Y T F} R_{T F}={ }^{Y T F} R_{G P S F}$. At each time instant, the rotation of the trailer with respect to the 737 world frame can be computed as:

$$
{ }^{W F} R_{T F}={ }^{W F} R_{\text {ITF }}{ }^{I T F} R_{\text {YTF }}{ }^{\text {YTF }} R_{A F}
$$

739 The position of trailer frame expressed in the world frame as:

740

$$
{ }^{W F} \mathbf{p}_{T F}={ }^{W F} H_{G P S F}{ }^{G P S F} \mathbf{p}_{T F}=\left[\begin{array}{ccc}
{ }^{W F} R_{T F} & { }^{W F} \mathbf{p}_{G P S F} \\
0 & 0 & 0
\end{array}\right.
$$

741 Now, at each time instant, the 3D georeferenced homogeneous position of each trailer beacon 742 antenna expressed in the world frame $\left({ }^{W F} \mathbf{b}_{j}=\left[b x_{j} b y_{j} b z_{j} 1\right]^{T}\right)$ can be computed using the following 743 equation:

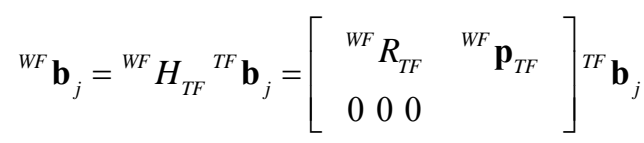

